\title{
Long Non-coding RNAs as Promising Therapeutic Approach in Ischemic Stroke: a Comprehensive Review
}

\author{
Marta Wolska $^{1}$ (D) Joanna Jarosz-Popek ${ }^{1}$ (D) - Eva Junger ${ }^{1}$ (D) - Zofia Wicik ${ }^{1,2}$ (D) Tahmina Porshoor $^{1}$ (D) \\ Lucia Sharif $^{1}$ (D) - Pamela Czajka ${ }^{1}$ (D) $\cdot$ Marek Postula $^{1}$ (D) - Dagmara Mirowska-Guzel ${ }^{1}$ (D) - Anna Czlonkowska ${ }^{1,3}$ (D) \\ Ceren Eyileten ${ }^{1}$ iD
}

Received: 22 September 2020 / Accepted: 9 November 2020 / Published online: 24 November 2020

(C) The Author(s) 2020

\begin{abstract}
In recent years, ischemic stroke (IS) has been one of the major causes of disability and mortality worldwide. The general mechanism of IS is based on reduced blood supply to neuronal tissue, resulting in neuronal cell damage by various pathological reactions. One of the main techniques for acute IS treatment entails advanced surgical approaches for restoration of cerebral blood supply but this is often associated with secondary brain injury, also known as ischemic reperfusion injury (I/R injury). Many researches have come to emphasize the significant role of long non-coding RNAs (lncRNAs) in IS, especially in I/R injury and their potential as therapeutic approaches. LncRNAs are non-protein transcripts that are able to regulate cellular processes and gene expression. Further, lncRNAs have been shown to be involved in neuronal signaling pathways. Several lncRNAs are recognized as key factors in the physiological and pathological processes of IS. In this review, we discuss the role of lncRNAs in neuronal injury mechanisms and their association with brain neuroprotection. Moreover, we identify the lncRNAs that show the greatest potential as novel therapeutic approaches in IS, which therefore merit further investigation in preclinical research.
\end{abstract}

Keywords Non-coding RNA · lncRNA · Stroke · Treatment · Ischemia/reperfusion · Therapeutic approach · Novel therapy

$\begin{aligned} & \text { Abbreviations } \\ & \text { 15-LOX1 }\end{aligned}$
AIM2
$\begin{aligned} & \text { 15-Lipoxygenase 1 } \\ & \text { Absent in melanoma } 2\end{aligned}$
- Acute injuries to CNS, including ischemic stroke, significantly alter
long non-coding RNA expression and function.
- Long non-coding RNAs may have potential as novel therapeutics in
ischemic stroke.

Ceren Eyileten

ceyileten@wum.edu.pl

Marta Wolska

wolska.marta@op.pl

Joanna Jarosz-Popek

j.jaroszpopek@gmail.com

Eva Junger

evamjunger@gmail.com

Zofia Wicik

zofiawicik@gmail.com

Tahmina Porshoor

tahmina.porshoor@gmail.com

Lucia Sharif

luciamsharif@gmail.com
AQP4 Aquaporin 4

ATG7 Autophagy-related 7

Bcl-2 B cell lymphoma 2
Pamela Czajka

czajka.pamela@gmail.com

Marek Postula

mpostula@wum.edu.pl

Dagmara Mirowska-Guzel

dmirowska@wum.edu.pl

Anna Czlonkowska

czlonkow@ipin.edu.pl

1 Department of Experimental and Clinical Pharmacology, Medical University of Warsaw, Center for Preclinical Research and Technology CEPT, Banacha 1B str., Warsaw, 02-097 Warsaw, Poland

2 Centro de Matemática, Computação e Cognição, Universidade Federal do ABC, Sao Paulo, Brazil

3 2nd Department of Neurology, Institute of Psychiatry and Neurology, 02-957 Warsaw, Poland 


\begin{tabular}{|c|c|c|c|}
\hline bFGF & Basic fibroblast growth factor & pSTAT3 & $\begin{array}{l}\text { Phosphorylation of signal transducer and } \\
\text { activator of transcription } 3\end{array}$ \\
\hline BDNF & Brain-derived neurotrophic factor & KCNO1OT1 & Potassium voltage-gated channel subfam- \\
\hline CASP3 & Caspase 3 & & ily Q member1 opposite stand 1 \\
\hline \multirow[t]{2}{*}{ СаMKII $\delta$} & Calcium/calmodulin-dependent protein & Q PCR & Quantitative polymerase chain reaction \\
\hline & kinase II $\delta$ & ROS & Reactive oxygen species \\
\hline CAMK2D & $\begin{array}{l}\text { Calcium/calmodulin-dependent protein } \\
\text { kinase II delta }\end{array}$ & RMST & $\begin{array}{l}\text { Rhabdomyosarcoma } 2 \text {-associated } \\
\text { transcript }\end{array}$ \\
\hline \multirow[t]{2}{*}{ CaMKII } & Calcium/calmodulin-dependent protein & Rock2 & Rho-associated protein kinase 2 \\
\hline & kinase II & Rian & RNA imprinted and accumulated in \\
\hline C2dat1 & CAMK2D-associated transcript & & nucleus \\
\hline CHRF & Cardiac hypertrophy-related factor & SIRT1 & Sirtuin 1 \\
\hline ceRNA & Competing endogenous RNA & siRNA & Small interfering RNA \\
\hline DAPK1 & Death-associated protein kinase 1 & SOX2 & Sex-determining region Y-box 2 \\
\hline DNMT3B & DNA methyltransferase 3B & SOX6 & Sex-determining region Y-box 6 \\
\hline \multirow[t]{2}{*}{ DUSP5-ERK1/2 } & Dual-specificity phosphatase & SNHG12 & Small nucleolar RNA host gene 12 \\
\hline & 5-extracellular signal-regulated kinase $1 / 2$ & $\mathrm{TNF} \alpha$ & Tumor necrosis factor $\alpha$ \\
\hline $\mathrm{EC}$ & Endothelial cells & TRAF & TNF receptor-associated factor \\
\hline FOXO3 & Forkhead box O3 & TUG1 & Taurine-upregulated gene 1 \\
\hline FOXO1 & Forkhead box protein $\mathrm{O} 1$ & ULK2 & Unc-51 like autophagy activating \\
\hline GATA3 & GATA binding protein 3 & & kinase 2 \\
\hline GAS5 & Growth arrest-specific transcript 5 & VEGFA & Vascular endothelial growth factor-A \\
\hline \multirow[t]{2}{*}{ HBMECs } & Human brain microvascular endothelial & VEGF & Vascular endothelial growth factor \\
\hline & cells & Oprm1 & $\mu$-opioid receptor 1 \\
\hline
\end{tabular}

HIF $2 \alpha \quad$ Hypoxia-inducible factor $2 \alpha$

IL-1 Interleukin-1

IL-18 Interleukin-18

IL-1 $\quad$ Interleukin-1 $\beta$

IL-6 Interleukin-6

IS Ischemic stroke

$\mathrm{I} / \mathrm{R} \quad$ Ischemic/reperfusion

LC3 Light chain 3 I (cytosol)

LC3 II Light chain 3 II (membrane)

lncRNAs Long non-coding RNAs

MALAT1 Metastasis-associated lung adenocarcinoma transcript 1

MEG3 Maternally expressed gene 3

MSCs Mesenchymal stem cells

miRNAs miRs MicroRNAs

MCAO Middle cerebral artery occlusion

MAP4K4 Mitogen-activated protein kinase kinase

kinase kinase 4

MCP-1 Monocyte chemoattractant protein-1

N2a Neuro2a

NMDA N-methyl-D-aspartate

NGF Nerve growth factor

$\mathrm{NF}-\kappa \mathrm{B} \quad$ Nuclear factor kappa B

NR3C2 Nuclear receptor subfamily 3 group $C$ member 2

OGD/R Oxygen-glucose deprivation/reperfusion

$\mathrm{PGC} 1 \alpha \quad$ Peroxisome proliferator-activated receptor gamma coactivator $1 \alpha$

PDCD4 Programmed cell death protein 4

\section{Introduction}

Ischemic stroke (IS), which constitutes more than $80 \%$ of strokes, generally results from reduced blood supply to cerebral tissue resulting in a number of pathological reactions such as oxidative stress, inflammation, and neuronal cell death [1]. Since IS is one of the major causes of disability worldwide, and since pharmacological approaches have generally failed in translation, there is a genuine requirement for novel therapeutic approaches. Previous researches have focused on preventing IS among those at risk, reducing severity, and promoting neurogenesis and neuronal recovery after IS. Early restoration of cerebral blood perfusion in ischemic tissues plays a pivotal role in IS treatment in order to minimize the severity of brain injury and neurological impairment [2]. Nevertheless, IS often leads to a secondary brain injury called ischemic/reperfusion (I/R) injury. The precise underlying mechanism of this injury remains unknown; however, many researchers have come to emphasize the role of inflammation, autophagy, and apoptosis as important contributors [3]. Moreover, vascular neural networks, especially brain microvascular endothelial cells (BMECs), are also prone to reperfusion damage. The I/R injury of BMECs leads to blood-brain barrier disruption, furthers brain injury, and is associated with poor prognosis in IS patients [4].

Long non-coding RNAs (IncRNAs) are a class of nonprotein transcripts which are greater than 200 nucleotides in 
length. LncRNAs play a crucial role in various cellular processes, including differentiation, apoptosis, as well as regulation of genes expression $[2,5]$. LncRNA alterations are often associated with a dysregulation of signaling pathways that control multiple neuronal, physiological, and pathophysiological processes. Additionally, lncRNAs can act as competing endogenous RNAs (ceRNA) of microRNAs (miRNAs) by binding to them, thereby reducing their regulatory effects on mRNA. MiRNA-mRNA interactions play an important role in the pathogenesis, diagnosis, and treatment of several diseases, including cardiovascular diseases, diabetes, as well as IS [1, 6-10]. LncRNAs, due to their broad range of action, can be targeted by various approaches either in the nucleus or in the cytoplasm. Firstly, small interfering RNA (siRNA), which activates the RNA-induced silencing complex, allows for post transcriptional RNA degradation. A similar degradation effect but with a different mechanism is achieved by antisense oligonucleotides. Besides, steric blockage of the promoter as well as techniques affecting the genome allows for lncRNAs gene regulation. RNA binding small molecules or morpholino oligonucleotides allow for RNA-protein interaction inhibition [11]. Nevertheless, there are many limitations associated with targeting lncRNAs as therapeutics in diseases, including crossing cellular plasma membrane and immune system response to foreign RNAs, resulting in a limited number of studies in this area.

In this review, we focus on IncRNAs that are able to regulate key factors involved in I/R injury such as calcium overload or glutamate toxicity. We present the relationship between lncRNAs and pathological processes that can contribute to ischemic injury exacerbations such as autophagy, inflammation, and oxidative stress. Moreover, we discuss lncRNAs which are involved in neuroprotection mechanisms and up-to-date knowledge regarding lncRNAs as promising therapeutic approaches in IS (Fig. 2).

\section{Article Search Process}

Electronic databases Pubmed and Scopus were searched between May 2020 and June 2020 and original studies were reviewed to evaluate the role of circulating lncRNAs in IS. Review articles and meta-analysis were incorporated in this as well as their secondary references for possible inclusion. Titles and abstracts were screened by two independent operators. The following search syntax was used: "Search ("long non coding RNAs" [MeSH Terms] or "IncRNA" [MeSH Terms] or "long non-coding RNA" [MeSH Terms] or "circulating lncRNA" [MeSH Terms] or "circulating long non coding RNA" [MeSH Terms]) and ("treatment" [MeSH Terms] or "therapeutic" [All Fields]) and ("ischemic stroke" [MeSH Terms] or "ischemia" [MeSH Terms] or "stroke" [MeSH Terms] or "ischaemic stroke" [MeSH Terms] or "ischaemia" [All Fields])" (Fig. 1).

\section{LncRNAs Regulating Glutamate Excitotoxicity in I/R Injury as a Key Factor in IS Severity and Neurological Impairment}

The precise underlying mechanisms involved in the pathophysiology of cerebral I/R injury remain relatively undiscovered. Nevertheless, lncRNAs which are able to modulate reperfusion injury, associated with enhanced neuronal cell death and hemorrhagic transformation, seem to play a significant role in IS treatment [12]. Studies showed that ischemic injury leads to excessive glutamate release which activates Nmethyl-D-aspartate (NMDA) receptors and causes an excessive calcium influx in neurons. Overstimulation of NMDA receptors by glutamate excitotoxicity is one of the trigger factors in initiating neuronal cell apoptosis [13]. Moreover, cerebral blood reperfusion of ischemic areas leads to enhanced calcium overload and further brain tissue damage. Excessive calcium accumulation results in the activation of calcium/ calmodulin-dependent protein kinase II (CaMKII), a family of multifunctional serine/threonine kinases involved in IS pathogenesis [14].

\section{LncRNA C2dat1}

Previous studies showed that CaMKII can be regulated by IncRNAs. One of the reports showed that the lncRNA CAMK2D-associated transcript (C2dat1) is able to regulate CaMKII $\delta$, a CaMKII isoform, by targeting CAMK2D [14]. The levels of lncRNA C2dat1 and CAMK2D were upregulated both in vivo and in vitro models of IS. Moreover, CaMKII $\delta$ was upregulated in the peri-infarct region, but was downregulated in the ischemic core. C2dat1 inhibition caused increased neuronal death and decreased levels of both CAMK2D and CaMII $\delta$. Prolonged inhibition of CaMII promoted neuronal apoptosis by increasing the glutamate toxicity vulnerability, whereas short-term inhibition protected against glutamate toxicity. Importantly, C2dat1 promoted neuronal survival via activation of nuclear factor kappa B (NF- $\mathrm{KB}$ ) signaling cascade, which may suggest $\mathrm{C} 2$ dat1 is a promising therapeutic approach for ischemia [14]. On the other hand, another report showed that the inhibition of CaMKII can prevent $30-70 \%$ of ischemia-induced neuronal death [15]. These discrepancies are probably caused by the fact that chronic inactivation of CaMKII increases the susceptibility of neurons to glutamate toxicity, whereas acute inactivation protects against glutamate, hypoxia, and hypoglycemia [16, 17]. Moreover, upregulation of CaMKII $\delta$ in the peri-infarct region and its downregulation in ischemic core could be explained by the fact that $C A M K 2 D$ is inactivated in a time- and locationdependent manner and the neurological deficit correlates with the rate of CAMK2D inactivation [14]. Consequently, early treatment with lncRNA C2dat1 can reduce the severity of 
Fig. 1 Article selection flowchart. The figure schematically depicts the article selection process, from literature search, through the screening, up to the final assessment of eligibility
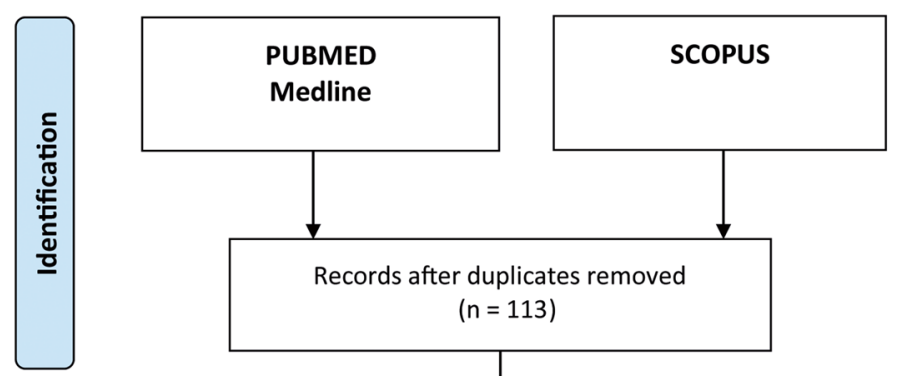

Records after duplicates removed $(n=113)$
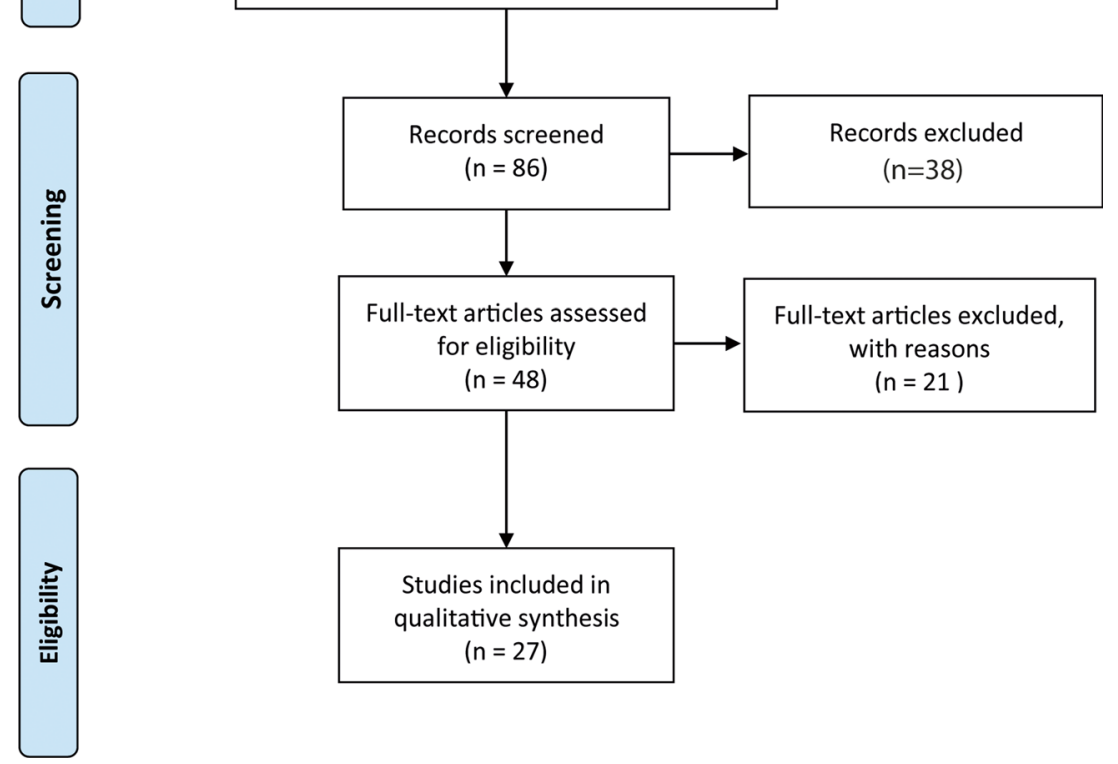

Sies included in ( $n=27$

neurological impairment after IS and should be further investigated in preclinical research (Fig. 3a).

\section{LncRNA AK038897}

Another lncRNA that may influence calcium homeostasis in IS is AK038897. AK038897 acts like a sponge by binding to miR-26a-5p and thus interfering with its target interactions. $D A P K 1$, a serine/threonine kinase, was determined as a target gene of miR-26a-5p. DAPK1 can be regulated by calcium/ calmodulin and is associated with excessive calcium influx through glutamate release, leading to mitochondrial dysfunction and neuronal membrane folding. AK038897 silencing could diminish DAPK1 levels via miR-26a-5p upregulation, contributing to protection against I/R injury. This observation was confirmed in vivo, as AK038897 knockdown resulted in reduced infarcted area and neurological impairment $[18,19]$. As previous studies reported that DAPK1 deletion prevents calcium overload and protects against ischemic neuronal death in IS [20], downregulation of DAPK1 by AK038897 can potentially protect against $\mathrm{I} / \mathrm{R}$ injury. Additionally, the transfection of DAPK1 caused more severe cerebral tissue damage in the IS mice model compared to the sham group $[13,18]$. Thus, these findings indicate that lncRNAs are able to regulate $D A P K 1$ and can be promising therapeutic approaches in IS.
Collectively, mentioned studies emphasized the role of mitochondrial dysfunction, excessive glutamate, and calcium accumulation in neuronal injury, indicating that lncRNAs which are capable of protecting mitochondrial membranes and controlling glutamate receptors and calcium channels can be promising therapeutic approaches in IS (Table 1).

\section{LncRNAs Involved in Signaling Pathways Responsible for Neuronal Cell Death}

Rising evidence confirms the crucial role of IncRNAs which acts as a sponge by binding to miRNAs and thus they are able to target multiple neural pathways. Hence, the main objective is to identify and understand those axes and find a proper way to alter its pathological effects in ischemic tissue.

\section{LncRNA GAS5}

The role of lncRNA GAS5 and its relationship with MAP4K4 gene was investigated by several experimental strategies. Overexpression of MAP $4 K 4$ reduced the apoptotic rate of oxygen-glucose deprivation/reperfusion $(\mathrm{OGD} / \mathrm{R}$, an in vitro model of IS)-induced neurons. In silico analysis predicted that lncRNA GAS5 interacts with MAP4K4. Moreover, DNMT3B was identified as a direct target gene of GAS5. LncRNA 


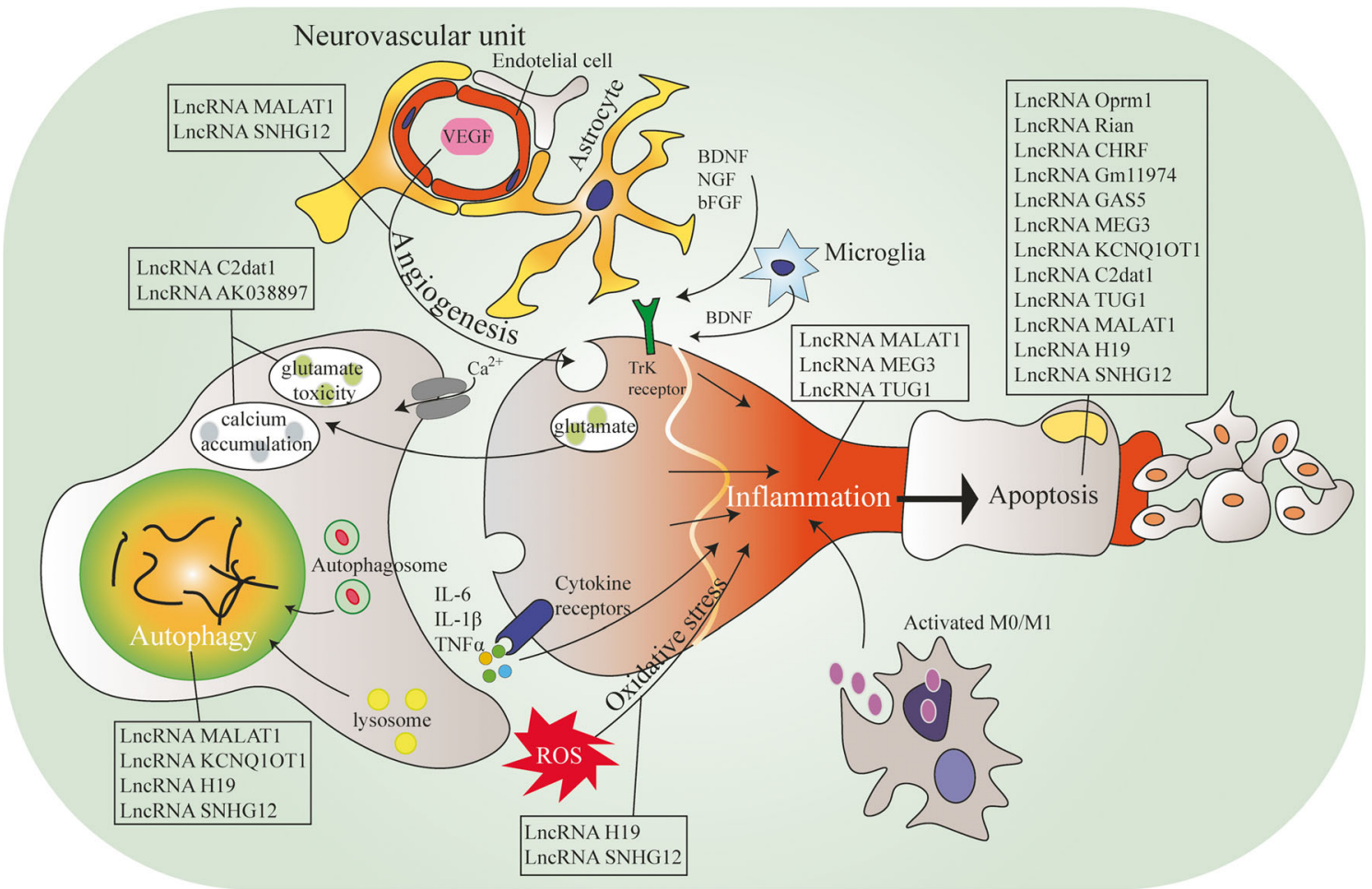

Fig. 2 The role of lncRNAs in the pathophysiology of neurodegeneration. Abbreviations: BDNF, brain-derived neurotrophic factor; bFGF, basic fibroblast growth factor; C2dat1, CAMK2Dassociated transcript; CHRF, cardiac hypertrophy-related factor; GAS5, growth arrest-specific transcript 5; IL-1 $\beta$, interleukin-1 $\beta$; IL-6, interleukin-6; KCNQ1OT1, potassium voltage-gated channel subfamily Q member1 opposite stand 1; MALAT1, metastasis-associated lung

GAS5 administration downregulated MAP4K4 expression through DNMT3B recruitment in mice primary cortical neurons. Furthermore, overexpression of lncRNA GAS5 increased the neuronal apoptosis rate. Additionally, lncRNA GAS5 administration resulted in a larger area of cerebral infarction and exacerbated neurological test results in animal models. Consequently, this study indicated that the inhibition of GAS5 in IS could potentially reduce neuronal apoptosis, reduce infarct size and improve neurological functioning via the MAP4K4/DNMT3B axis [40] (Fig. 3b).

\section{LncRNA N1LR}

Genome-wide lncRNA expression analysis revealed that lncRNA N1LR is significantly altered during I/R injury. The expression of N1LR was increased after mild ischemic injury, but reduced after more severe ischemic injury. Additionally, N1LR was mainly located in the cytoplasm in undamaged tissue, however after I/R injury N1LR was mostly accumulated in the nucleus. Overexpression of IncRNA N1LR accelerated cell cycle progression, promoted proliferation, and inhibited apoptosis after OGD/R injury (in vitro model). Moreover, overexpression of N1LR resulted in reduced infarct volume and neurological deficits, while silencing caused adenocarcinoma transcript 1; MEG3, maternally expressed gene 3; M0/ M1, microglia non-activated/pro-inflammatory; NGF, nerve growth factor; Rian, RNA imprinted and accumulated in nucleus; SNHG12, small nucleolar RNA host gene 12; TNF $\alpha$, TNF receptor-associated factor; TrK, tropomyosin receptor kinase; TUG1, taurine-upregulated gene 1; VEGF, vascular endothelial growth factor

the opposite effect in the animal model. Furthermore, N1LR administration significantly reduced neuronal apoptosis. Importantly, N1LR inhibited phosphorylation of the p53 protein, suggesting that the protective effect of IncRNA N1LR against $\mathrm{I} / \mathrm{R}$ injury may be due to the blockage of $\mathrm{p} 53$ phosphorylation [35].

Overall, more and more lncRNAs are being characterized functionally in a different neuronal cell context. Previous studies have shown that lncRNAs, as a part of complex signaling pathways, are able to regulate neuronal apoptosis, inflammation, and IS severity. Notably, the complexity of those axes allows for their modification at various stages. Further studies are needed on the cellular effects that might be caused by targeting lncRNAs as a part of the multifunctional signaling pathways involved in IS (Table 1).

\section{LncRNA Oprm1}

The role of lncRNA Oprm1 in cerebral I/R injury was studied both in vitro and in vivo models of IS. Following the reperfusion time, the lncRNA Oprm1 expression was decreased in vitro. The overexpression of Oprm1 decreased the apoptosis rate as well as reduced the infarct size and improved the neurological functioning of $\mathrm{I} / \mathrm{R}$ model experimental animals. 


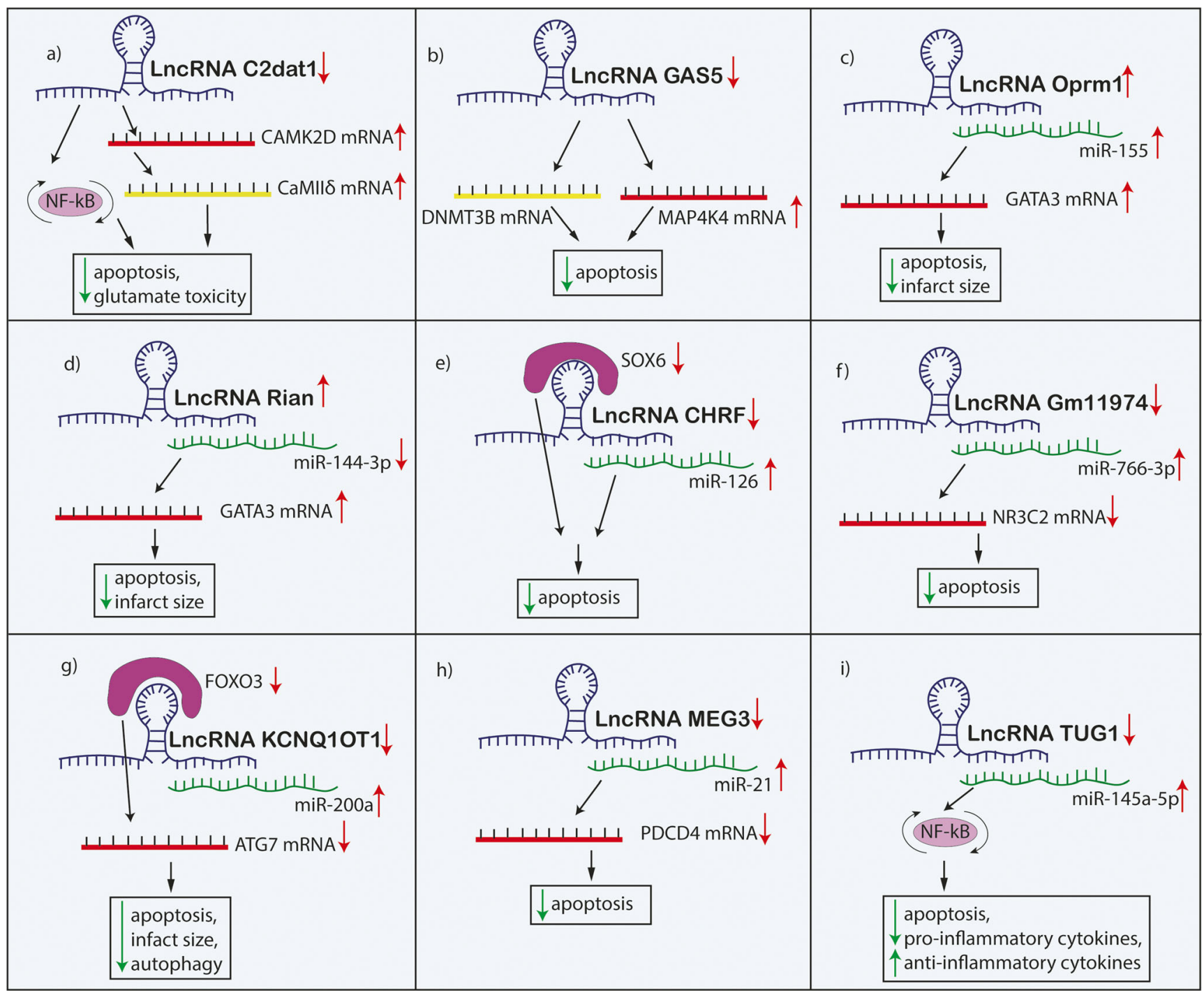

Fig. 3 The mechanisms by which IncRNAs contribute to the neuronal cell death process in ischemic stroke. Abbreviations: Oprm1, $\mu$-opioid receptor 1; GATA3, GATA binding protein 3; Rian, RNA imprinted and accumulated in nucleus; CHRF; cardiac hypertrophy-related factor; SOX6, sex-determining region Y-box 6; NR3C2, nuclear receptor subfamily 3 group C member 2 ; GAS5, growth arrest-specific transcript 5 ; DNMT3B, DNA methyltransferase 3B; MAP4K4, mitogen-activated

As Oprm1 can directly target miR-155, whereas miR-155 can directly target GATA3, Oprm1 demonstrated a protective role by sponging miR-155, thus regulating GATA3 expression. GATA3 is involved in neuronal differentiation and survival of sympathetic progenitors and neurons in embryonic chromaffin cells in in vitro analysis [41]. Collectively, this study suggests the importance of the Oprm1/miR-155/GATA3 axis in neuronal cell death [6]. Importantly, not only Oprm1 treatment but also miR-155 alone treatment decreased the infarct size; moreover it also reduced the volume of hemorrhagic transformation of IS, suggesting that Oprm $1 / \mathrm{miR}-155$ axis can exert more potent protective effect than miR-155 alone treatment [30] (Fig. 3c). protein kinase kinase kinase kinase 4; MEG3, maternally expressed gene 3; PDCD4, programmed cell death 4; KCNQ1OT1, potassium voltagegated channel subfamily Q member1 opposite stand 1; FOXO3, forkhead box O3; ATG7, autophagy-related 7; C2dat1, CAMK2D-associated transcript; CAMK2D, calcium/calmodulin-dependent protein kinase II; $\mathrm{CaMII} \delta$, calcium/calmodulin-dependent protein kinase II $\delta$; NF- $k \mathrm{~B}$, nuclear factor kappa B; TUG1, taurine-upregulated gene 1

\section{LncRNA Rian}

Similarly, the role of lncRNA Rian and its relation with miR144-3p levels and GATA3 were studied in IS experimental models. Rian, as well as GATA3, were downregulated in both in vitro and in vivo models, whereas miR-144-3p was upregulated. Overexpression of Rian reversed miR-144-3p-induced cerebral ischemic injury in mice. Moreover, overexpression of IncRNA Rian reduced the neuronal apoptosis and infarct size and improved the neurological score. GATA3 was identified as a target gene of miR-144-3p and was involved in the miR144-3p-mediated injury mechanism. Importantly, lncRNA Rian also regulates GATA3 and overturns the miR-144-3p- 


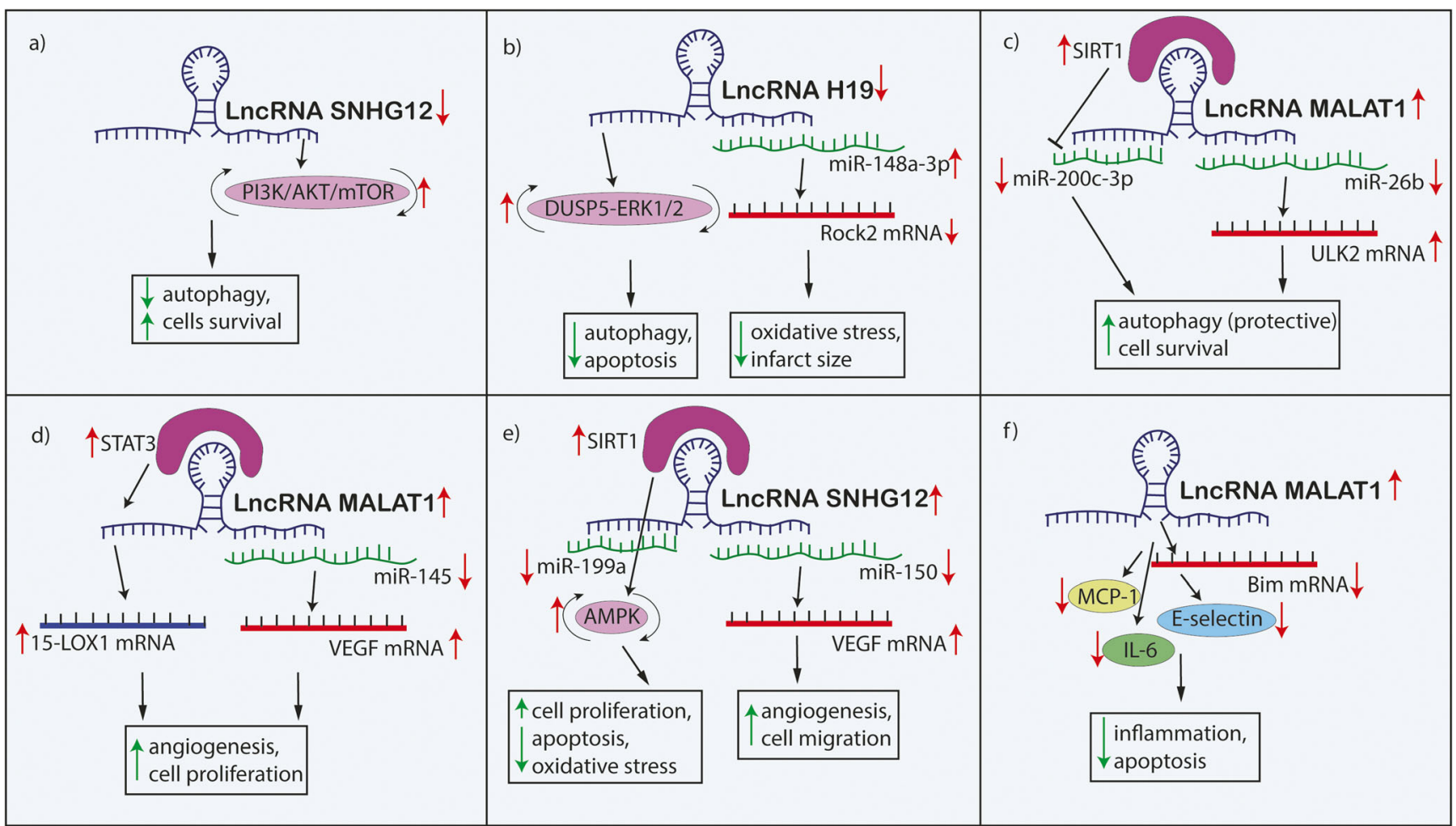

Fig. 4 The mechanism of lncRNAs MALAT1, H19, and SNHG12 in ischemic stroke pathogenesis. Abbreviations: MALAT1, metastasisassociated lung adenocarcinoma transcript 1; ULK2, Unc-51-like autophagy activating kinase 2; 15-LOX1, 15-lipoxygenase 1; VEGF, vascular endothelial growth factor; MCP-1, monocyte chemoattractant protein-1; IL-6, interleukin-6; DUSP5-ERK1/2, dual-specificity phosphatase 5-

induced suppression of GATA3. Collectively, lncRNA Rian can potentially suppress miR-144-3p-mediated neuronal loss via Rian/miR-144-3p/GATA3 axis. Therefore, it can be treated as an important therapeutic approach and should be further investigated in preclinical research [21] (Fig. 3d).

\section{LnCRNA CHRF}

LncRNA CHRF was found to play an important role in the processes of cardiac dysfunction and in the regulation of myocardiocyte death after reperfusion [42]. Besides, the importance of CHRF on I/R injury in IS was also determined. LncRNA CHRF was found to be upregulated after I/R injury and could directly bind to miR-126, whereas miR-126 was downregulated in I/R injury. Moreover, CHRF can directly target SOX6 which is associated with diverse apoptosis mechanisms and thus can modulate ischemic cell apoptosis. Knockdown of CHRF significantly increased miR-126 and decreased SOX6 expression. Thus, inhibition of CHRF can reduce neuronal damage and neurological impairment by targeting the miR-126/SOX6 cascade [43]. It is important to note that miR-126 was intensively studied in the context of platelet function in patients with hyper platelet activity and growing evidence showed that miR-126 can be a promising extracellular signal-regulated kinase 1/2; Rock2, Rho-associated protein kinase 2; SIRT1, sirtuin 1; SNHG12, small nucleolar RNA host gene 12; AMPK, AMP-activated protein kinase; PI3K, phosphoinositide 3kinase; AKT, protein kinase $\mathrm{B}$; mTOR, the mammalian target of rapamycin

biomarker in platelet activation [8, 9, 44]. Platelet activation and aggregation are critical in the pathogenesis of IS. Patients with IS exhibit hyper-reactive platelets compared to healthy individuals [45]. Therefore, future analysis should aim to analyze the importance of $\mathrm{CHRF} / \mathrm{miR}-126$ sponging regarding platelet activation in patients with IS (Fig. 3e).

\section{LncRNA Gm11974}

The function of another lncRNA Gm11974 was evaluated in the context of neuronal cell death in an IS in vitro model. Knockdown of Gm1 1974 caused a significant decrease in cell death rate and apoptosis as well as increased cell viability and protected mitochondrial membrane potential. Furthermore, the inhibition of Gm1 1974 increased the expression of miR$766-3 p$ in vitro, demonstrating that Gm1 1974 could potentially negatively regulate miR-766-3p. Moreover, NR3C2 was negatively regulated by miR-766-3p. Knockdown of IncRNA Gm11974 decreased cell apoptosis through miR766-3p upregulation, which antagonized NR3C2, demonstrating the role of Gm11974/miR-766-3p/NR3C2 axis in I/R injury. Consequently, these results indicate that silencing of Gm11974 may protect against cerebral reperfusion injury; 


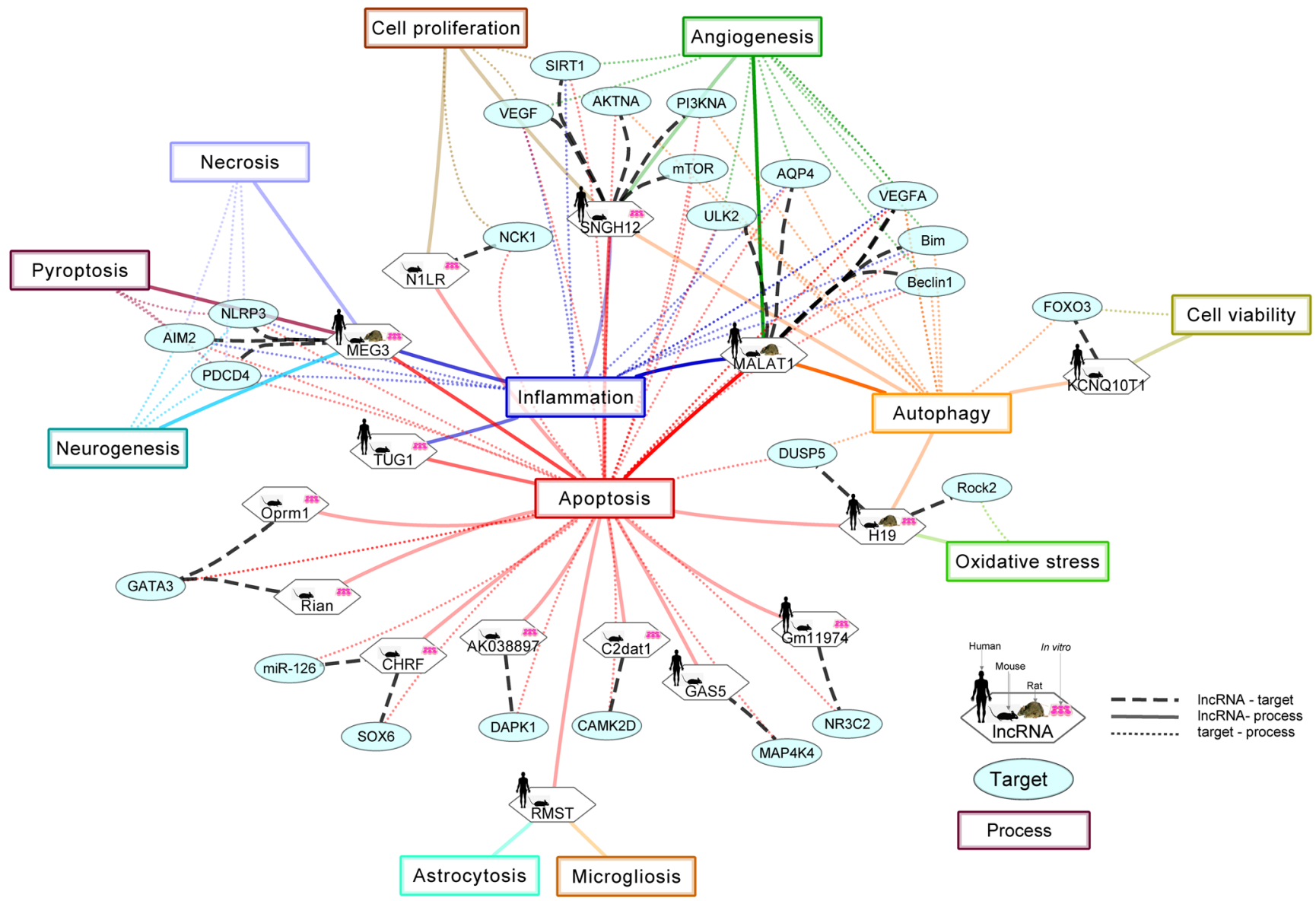

Fig. 5 Summary network graph showing information gained from studies investigating lncRNAs as potential novel therapeutic approaches in ischemic stroke

thus this lncRNA should be further investigated for clinical application as a therapeutic approach in IS [46] (Fig. 3f).

\section{LnCRNA MALAT1}

LncRNA MALAT1 and its association with AQP4 in cerebral $\mathrm{I} / \mathrm{R}$ injury was determined in OGD/R astrocyte cell and middle cerebral artery occlusion (MCAO) mouse models. MALAT1 expression was upregulated in both models. Knockdown of MALAT1 increased the survival of astrocytes and decreased the apoptotic rate. Additionally, knockdown of $A Q P 4$, a transmembrane protein associated with brain edema and apoptosis, caused a decrease in cytotoxicity and reduced apoptosis of astrocytes. Moreover, cell damage was alleviated after the AQP4 knockdown. As it was determined that MALAT1 can act as ceRNA for miR-145, MALAT1 positively regulated $A Q P 4$ via miR-145 downregulation. Altogether, the study validated that MALAT1 silencing protects against cerebral I/ $\mathrm{R}$ injury by regulating the miR-145/AQP4 axis [3].

To sum up, IncRNA Oprm1 and lncRNA Rian demonstrate protective properties against I/R injury through GATA3 modulation. Besides, IncRNA CHRF, MALAT1, and Gm11974 also present positive effects on pathological processes in $\mathrm{I} / \mathrm{R}$ injury. Consequently, all the mentioned lncRNAs show great potential as novel therapeutic approaches in IS and therefore merit further investigation in preclinical research (Table 1).

\section{Ischemia/Reperfusion Injury Exacerbating Factors as Therapeutic Approaches}

Following cerebrovascular thrombosis, reperfusion of tissues by the restoration of adequate oxygen supply may cause further damage to brain tissue and should be distinct from the injury that is caused by ischemia. Changes that occur in brain tissue under deprivation of oxygen and other nutrients cause oxidative and inflammatory damage of neuronal cells [47]. This oxidative stress is likely to contribute to autophagy activation, which determines subsequent processes in the $\mathrm{I} / \mathrm{R}$ injury region [48]. The significance of autophagy in IS will be broadly described in this section, as the final effect on neurons is highly dependent on the phase of reperfusion. Previous evidence suggests that autophagy, angiogenesis, as well as oxidative stress have a critical effect on $\mathrm{I} / \mathrm{R}$ injury and can be moderated by lncRNAs [49]. Exploring lncRNAs in the abovementioned processes may help to understand the 


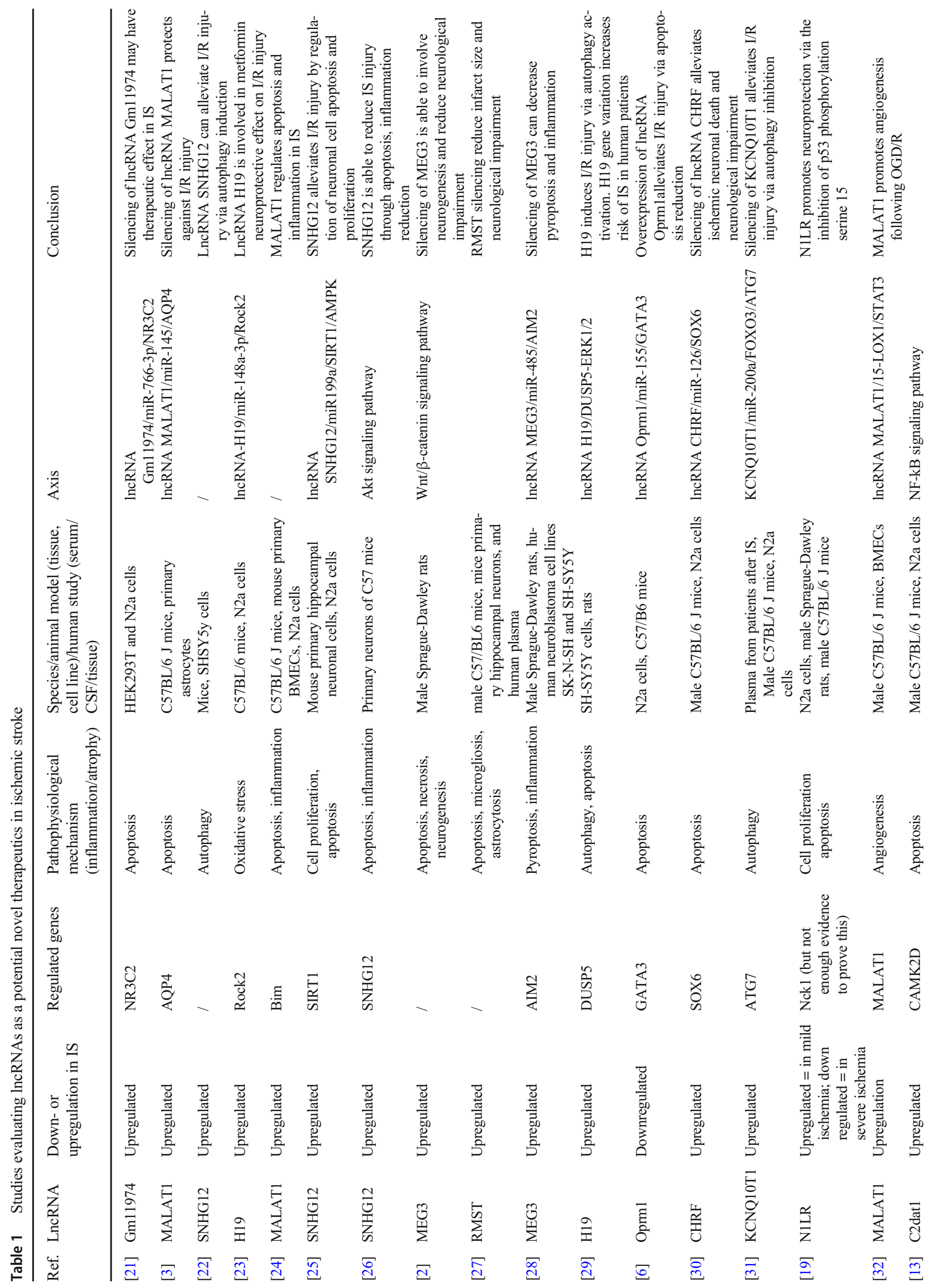




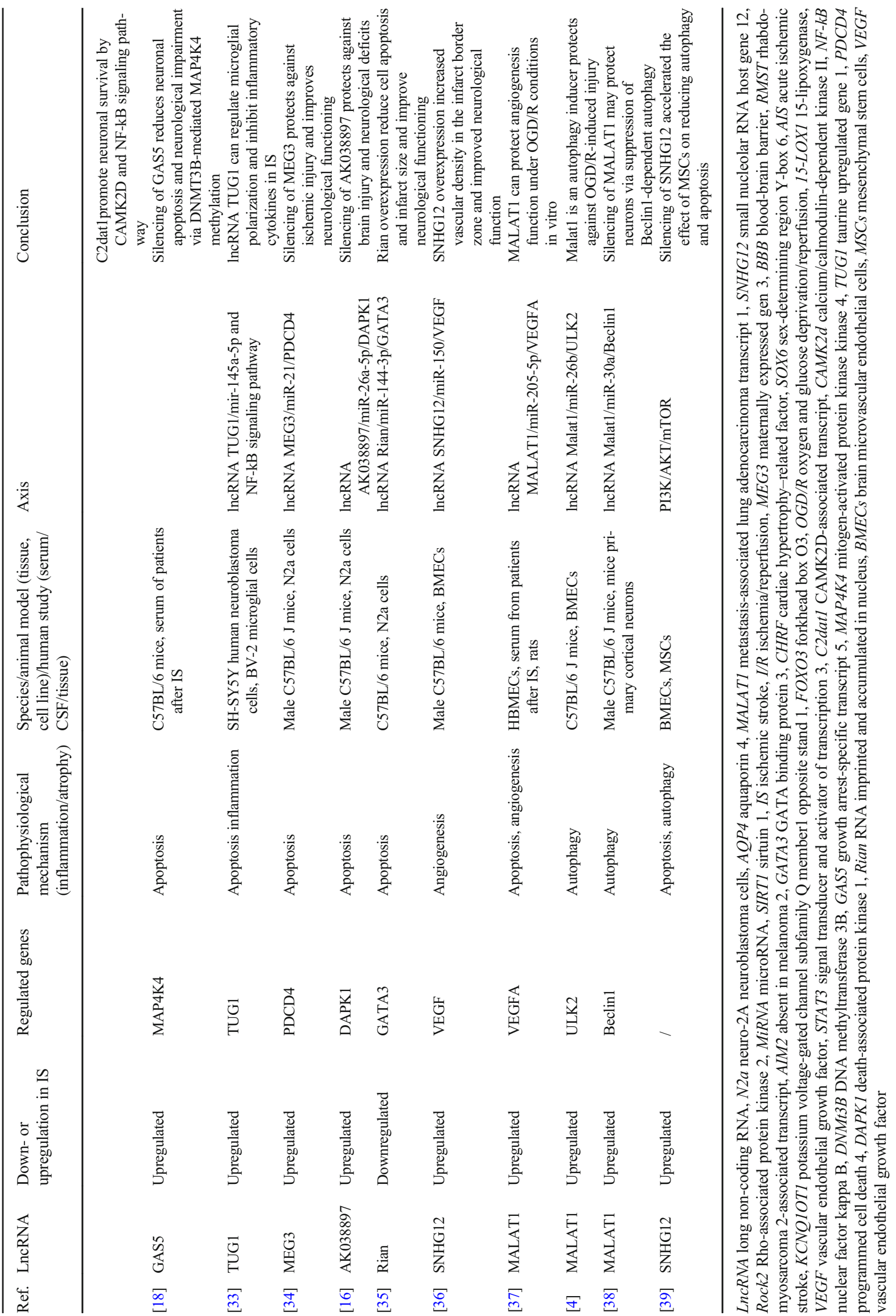


pathomechanisms involved in IS and thus recovery networks induced after ischemia.

\section{Autophagy}

Autophagy, as a lysosomal degradation pathway, is responsible for the recycling of damaged and aged cellular components and is crucial for neuronal hemostasis [50]. As described by Wang et al., the activation of the autophagy pathway in the brain upon ischemic stimuli can be a double-edged sword for neural survival after IS. To date, some studies have demonstrated a protective role of autophagy in IS while others have shown damaging effects [51]. Importantly, several authors have underlined that autophagy can be neuroprotective during early stages of ischemia; however, prolonged ischemia leads to neurotoxic autophagy [52]. Besides, it was also hypothesized that autophagy can show protective effects during ischemia, whereas it can be detrimental during reperfusion period [22]. Autophagy activity might be measured using autophagyrelated gene expression assessment such as Beclin-1, LC3 I, and $L C 3 I I$ [39]. The role of IncRNAs in the regulation of autophagy has been extensively investigated in previous years; however, the majority of research concentrated on myocardial infarction and its reperfusion injury [29, 31, 53]. The association of lncRNAs and autophagy-related genes in IS remains unclear and should be further investigated.

\section{LncRNA SNHG12}

The expression of lncRNA SNHG12 was shown to be upregulated in cerebral I/R injury and associated with enhanced autophagy activation and increased neuronal cell survival both in in vitro and in vivo analysis [22, 54]. Additionally, the role of SNHG12 as a regulator of mesenchymal stem cell (MSC) function in I/R injury was also determined. Downregulation of SNHG12 was found to potentiate the ability of MSCs to reduce autophagy via $\mathrm{PI} 3 \mathrm{~K} / \mathrm{AKT} / \mathrm{mTOR}$ axis. In I/R injury treated with SNHG12-modified MSCs, autophagy, apoptosis, and infarcted regions were reduced [55]. Collectively, lncRNA SNHG12 might be an important regulator of autophagy in IS and possesses therapeutic potential in I/R injury. Nevertheless, as previous studies demonstrated, the neuroprotective role of IncRNA SNHG12 in IS can be achieved by both reduction and enhancement of autophagy (Fig. 4a).

\section{LncRNA H19}

LncRNA H19 was found to play an important role in stimulating autophagy in cerebral $\mathrm{I} / \mathrm{R}$ injury, as significant overexpression of IncRNA H19 and autophagy activation in cerebral tissue were observed in previous experiments. Further analysis showed that IncRNA H19 decreases cell viability via autophagy promotion through inhibition of the DUSP5-ERK1/2 pathway. LncRNA H19 inhibition resulted in reduced autophagy and neuronal cell apoptosis in in vitro analysis. Furthermore, the study revealed that $H 19$ gene variations are associated with increased risk of IS. Consequently, lncRNA H19 seems to play an important role in I/R injury via autophagy activation and its inhibition may have therapeutic effect on cerebral tissue in IS [56] (Fig. 4b).

\section{LncRNA KCNQ10T1}

Another lncRNA KCNQ1OT1 was upregulated in IS patients' plasma and was correlated with IS severity. Administration of KCNQ1OT1 inhibitor in mice decreased ATG7 (autophagyrelated gene) expression, which resulted in reduced infarct volume, neurological impairment, and inhibited autophagy. Similar results associated with autophagy were observed in vitro, but importantly, activated autophagy promoted neuronal apoptosis and could be inhibited by KCNQ1OT1 knockdown, suggesting its protective role. MiR-200a was detected as a direct target of KCNQ1OT1. MiR-200a can regulate FOXO3 expression, which is able to regulate $A T G 7$, and thus autophagy. Knockdown of KCNQ1OT1 increased miR-200a and decreased FOXO3 expression. Collectively, inhibition of KCNQ1OT1 is associated with reduced autophagy and therefore neuronal dysfunction through the miR-200a/FOXO3/ $A T G 7$ axis [57] (Fig. 3g).

As above mentioned, the role of autophagy in IS is unclear. There are more than 300 studies considering the role of autophagy in IS, some demonstrating autophagy inhibitors to induce a neuroprotective effect and reduce infarct size and apoptosis rate $[38,51,58]$, while others reported autophagy inhibition to result in increased neuronal cell apoptosis and neurological deficits $[38,51,58,59]$. Based on these reports, it can be hypothesized that lncRNAs enhance autophagy during early stages of ischemia, while reducing autophagy in prolonged ischemia and I/R injury. Therefore lncRNAs should be a target of future research investigating autophagy effects on ischemia.

\section{LncRNA MALAT1}

The role of lncRNA MALAT1 is broadly emphasized in various processes following IS, including autophagy regulation in the I/R injured brain area. To date, there are three studies that have investigated the relationship of MALAT1 and autophagy in IS. Overexpression of MALAT1 was able to enhance autophagy and improve the neuronal survival in an in vitro model of IS. The study revealed that MALAT1 acted as an endogenous sponge of miR-26b and downregulated its expression. Prediction analysis showed that $U L K 2$, autophagy-related gene, is a direct target gene of miR-26b. Overexpression of MALAT1 upregulated ULK2 via downregulation of miR-26b and increased autophagy rate and 
neuronal survival. Overall, the results suggest that MALAT1/ miR-26b/ULK2 axis promotes autophagy and survival [4]. Similarly, another study showed that MALAT1 was upregulated in the IS in vitro model and its overexpression was associated with enhanced autophagy and increased cell survival. Furthermore, MALAT1 reduced the expression of miR-200c$3 p$. MALAT1 directly binds to miR-200c-3p and cells that were treated with MALAT1 presented reduced apoptosis. Importantly, MALAT1 induces SIRT1 (an autophagy activator and neuroprotector factor) by blocking miR-200c-3p expression [32, 37] (Fig. 4c). Contrary to previous studies, Guo et al. reported that downregulation of MALAT1 reduced autophagy and thus promoted neuronal cell survival after IS both in in vitro and in vivo models. The study showed that silencing of MALAT1 upregulated miR-30a. Beclin-1, an autophagy biomarker, was a target gene of miR-30 and was negatively regulated by miR-30. Thus, silencing of MALAT1 suppressed Beclin-1-dependent autophagy via Beclin-1 downregulation [36]. As mentioned previously, the contrary results can be explained by the unclear role of autophagy in IS, as it might have either positive or negative effects on neuronal cells. Collectively, lncRNA MALAT1 regulation of autophagy in $\mathrm{I} / \mathrm{R}$ injury can promote both neuroprotection and neuronal death. Thus, further studies are needed in this topic to clarify the underlying mechanisms.

\section{Angiogenesis}

Neuronal angiogenesis is a multi-step process, involving the proliferation of human brain microvascular endothelial cells (HBMECs) which differentiate into tubular micro-vessels. Several reports have demonstrated the promotion of angiogenesis in specific brain regions after ischemic injury. Furthermore, there is a correlation between angiogenesis and improved neurological functioning after IS [60]. Even though the role of lncRNAs in angiogenesis after I/R injury remains unclear, there are few studies emphasizing the importance of MALAT1 and SNHG12 in the process of angiogenesis in IS.

\section{LncRNA MALAT1}

It was previously found that MALAT1 is upregulated in both in vitro and animal models as well as in patients with IS. MALAT1 downregulation was related to reduced EC proliferation, cell migration, and reduced CD31 expression (an angiogenesis-associated marker) leading to reduced angiogenesis capacity in vitro. Furthermore, MALAT1 knockdown reduced the levels of 15-LOX1, VEGF, the phosphorylation of STAT3 (important angiogenesis regulating factors) indicating that MALAT1 may not only control angiogenesis by ischemic stimuli but also via 15-LOX1/STAT3 signaling pathway [61]. Moreover, MALAT1 overexpression can indirectly increase the expression of VEGFA in an OGD/R model of HBMEC.
Similarly, the overexpression of MALAT1 was associated with increased angiogenesis and cell proliferation. Furthermore, it was also shown that MALAT1 acted as a ceRNA of miR-205-5p, which is able to upregulate VEGFA [62]. Consequently, these studies have confirmed that IncRNA MALAT1 is able to protect the angiogenesis function in ischemic conditions via the regulation of different miRNAs, which are henceforth able to upregulate pro-angiogenic factors (Fig. 4d).

\section{LncRNA SNHG12}

The role of lncRNA SNHG12 in angiogenesis after IS was determined. SNHG12 was upregulated in vitro and its overexpression was associated with increased angiogenesis and cell migration. Moreover, SNHG12 acted as a ceRNA of miR-150 and thus interfered with its target interactions. Downregulation of SNHG12 was able to enhance the tube formation (hallmark of neovascularization degree) and indirectly upregulated the level of VEGF, which was identified as a target gene of miR-150 [25]. Similarly, He et al. reported that the overexpression of miR-150 was associated with a decreased vascular density in the infarcted area, decreased tube formation, BMECs migration, and VEGF expression in in vivo analysis, confirming the previous results [23]. Thus, IncRNA SNHG12 also seems to have therapeutic potential in IS by regulating angiogenesis. As the number of studies are limited, further analyses are needed to confirm the therapeutic potential of MALAT1 and SNHG12 in IS (Fig. 4e).

\section{Excessive Oxidative Stress}

Reperfusion injury is also largely attributed to the excessive production of reactive oxygen species (ROS) in ischemic tissue [63]. Many studies underline the association of excessive oxidative stress and lncRNAs in I/R injury. Sirtuins, which can be regulated by lncRNAs, were shown to alleviate $I / R$ injury by protecting against cellular stress. SIRT1 in particular demonstrated beneficial effects against oxidative stress by activating FOXO1, $\mathrm{PGC} 1 \alpha$, and $\mathrm{HIF} 2 \alpha$ and by inhibiting the NF-kB transcription factor [64], which makes sirtuins important contributors against I/R injury.

\section{LncRNA SNHG12}

The effect of lncRNA SNHG12 on I/R injury and its association with sirtuins were studied in an in vitro model. Initially, upregulated SNHG12 expression was shown in primary neuronal cells and Neuro2a (N2a) cells after OGD/R. Knockdown of SNHG12 caused inhibition of cell proliferation and increased cell apoptosis. Importantly, SNHG12 downregulated miR-199a, which inhibits cell proliferation and induces cell apoptosis; thus, SNHG12 overexpression can contribute to the 
I/R injury reduction. Overexpression of miR-199a or knockdown of SNHG12 inhibited the expression of SIRT1, suggesting that SNHG12 upregulates SIRT1 by downregulation of miR-199a. Hence, miR-199 might be used as a biomarker to monitor the therapeutic response to SNHG12. SNHG12 can be an important regulatory factor of oxidative stress by upregulating SIRT1 through activation of the AMPK pathway and inhibiting miR-199a in neuronal cells with I/R injury [65] (Fig. 4e). On the other hand, previous clinical study showed that miR-199 was upregulated in patients with heart failure [66]. Thus, well-designed clinical studies are needed in order to confirm the impact of SNHG12/miR-199 sponging in ischemia.

\section{LncRNA H19}

Another study determined the protective effect of metformin in mouse brains after MCAO, as the treatment improved neurobehavioral function and decreased infarct volume. Metformin decreased oxidative stress both in the in vitro and in vivo analysis. Importantly, metformin inhibited lncRNA H19 expression and increased the expression of miR-148a3p. Rock 2 was determined as a target gene of miR-148a-3p, and upregulation of miR-148a-3p decreased Rock2 expression. Therefore, the neuroprotective effect of metformin against oxidative stress injury was observed through the lncRNA H19/miR-148a-3p/Rock2 axis [67].

Collectively, the studies showed that lncRNAs are potentially able to mediate oxidative stress injury in IS, especially via protein regulation such as sirtuins. Thus, lncRNA SNHG12 and H19 can contribute to reduced neurological impairment (Fig. 4b)

\section{Potentially Modifiable Factors Contributing to Recovery Following IS}

The human brain possesses the capacity of self-repair after injury. Neurogenesis is a process based on the formation of neuronal cells from neural stem cells and is present in specific brain regions. Various factors such as those belonging to neurotrophic factors may enhance the process. One of the neurotrophins, brain-derived neurotrophic factor (BDNF), has a well-documented ability to promote neuroplasticity and was previously described to facilitate post-stroke rehabilitation and recovery [27, 68-74]. Recent studies indicate that IS leads to prolonged production of new striatal neurons [75]. Another factor affecting recovery after IS, post-ischemic neuroinflammation, can be influenced by modifying various proinflammatory factors [76]. Thus, neurological recovery can be supported by modification of neurogenesis and neuroinflammatory processes.
Neuronal Plasticity and Neuronal Repair Mechanisms

\section{LncRNA RMST}

LncRNA RMST modulates neurogenesis by the regulation of neural cell fate decisions and neuronal differentiation. RMST co-regulates transcription factor SOX2 and is essential for the connection of SOX2 with its neurogenic target genes [77]. LncRNA RMST expression was found to be significantly increased in both in vitro and I/R animal models, as well with patients after IS. In vitro, RMST silencing caused decreased neuronal apoptosis and a partial reversibility of the OGD/R injury on the cell viability. In an animal model, RMST silencing caused a reduced infarction size and improved neurological function test results. Moreover, reduction of brain microgliosis and astrocytosis markers in the hippocampal region was also observed [78]. The influence of lncRNA RMST on astrocytosis and microgliosis processes is unclear. Previous studies reported that astrocytosis and microgliosis play key roles in neurological recovery, including scar formation and release of molecules promoting neuronal plasticity [79]. Nevertheless, in the acute phase of IS, astrocytosis and microgliosis have neuroprotective effects, while during the chronic phase, astrocytes may have both harmful and protective effects [80].

\section{LnCRNA MEG3}

Besides, lncRNA MEG3 plays an important role in nerve growth and neurological deficit after I/R injury. MEG3 was investigated on an animal model. In order to identify neurogenesis conditions, nerve growth factor (NGF) levels were determined, including BDNF, NGF, and basic fibroblast growth factor (bFGF). MEG3 overexpression was clinically observed as increased neurological impairment, larger infarct area, increased water content, increased blood-brain barrier permeability, neuronal apoptosis, and necrosis as well as upregulation of Wnt/B-catenin proteins, whereas MEG3 inhibitor administration resulted in opposite effects. Importantly, the treatment with MEG3 inhibitors resulted in increased BDNF, NGF, and bFGF levels. It is well known that BDNF, NGF, and $\mathrm{bFGF}$ are involved in neuronal repair processes, including axonal growth and proliferation of neuron progenitors $[27,28$, 34, 68-70, 73, 74, 81]. Thus, the results indicate that blocking of IncRNA MEG3 expression may stimulate nerve growth and neurogenesis by increasing NGFs and may decrease neurological impairment through the Wnt signaling pathway [2].

Overall, in order to identify the effective therapeutic approach, more attention should be paid to neurogenesis following IS as well as neuroprotection and reduction of postischemic neuronal inflammation. LncRNAs, which are able to influence the abovementioned processes, seem to be promising therapeutic approaches in IS (Table 1). 


\section{Post-Ischemic Neuronal Inflammation}

\section{LncRNA MEG3}

Inflammasomes are large complexes, formed in response to inflammatory stimuli, which contain various molecules such as caspases and are able to promote maturation of proinflammatory cytokines, i.e., interleukin-1 $\beta$ (IL-1 $\beta$ ) and interleukin-18 (IL-18) [82]. Apart from pro-inflammatory cytokines, studies showed that AIM2 inflammasomes play an important role in ischemic brain injury. The activation of AIM2 inflammasomes can potentially induce pyroptosis and inflammation in surrounding cells, increasing the injury area [24]. Due to these presumptions, IncRNA MEG3-mediated modulation of AIM2 inflammasome was investigated in IS. Firstly, it was determined that MEG3 acted as a sponge for miR-485 to suppress its expression. Moreover, MEG3 and AIM2 expressions were upregulated, whereas miR-485 was downregulated both in in vivo and in vitro analysis. Knockdown of MEG3 reduced the pyroptosis and inflammation by the upregulation of miR485 and the downregulation of the AIM2 inflammasome signaling, whereas miR-485 inhibitor reversed the effect [26]. Similarly, Yan et al. also observed that the inhibition of MEG3 improved neurological functioning by targeting miR21/PDCD4 axis [33] (Fig. 3h).

A handful of neuronal inflammasomes are broadly reported in literature as potential therapeutic approaches in IS [83, 84]; however, little is known about their association with lncRNAs. The AIM2 inflammasome regulated by lncRNA MEG3 is able to mediate neuronal pyroptosis, which is an inflammatory programmed cell death and is associated with membrane pore formation, cell lysis, and release of cell content. Overall, this suggests that IncRNA can potentially regulate inflammasome-mediated neuroinflammation in IS.

\section{LnCRNA MALAT1}

The effects of lncRNA MALAT1 were also studied in postischemic inflammation. Silencing of MALAT1 caused a significant increase in cerebral vascular endothelial cell death and increased CASP3 activity in vitro. Similarly, silencing of MALAT1 was associated with larger infarct volumes and more serious neurological deficits in response to ischemic injury in vivo. Moreover, silencing of MALAT1 drastically increased mRNA levels of pro-apoptotic factor Bim (a member of the Bcl-2 family) and induced pro-inflammatory cytokines, such as E-selectin, monocyte chemoattractant protein (MCP-1), and interleukin-6 (IL-6) after cerebral ischemia. Additionally, it was shown that MALAT1 is a direct target for both Bim and E-selectin. Ultimately, the study recognized novel functions of MALAT1, the regulation of apoptotic, and inflammatory responses in mouse cerebral endothelium after in vitro and in vivo cerebral ischemic insults [85] (Fig. 4f).

\section{LncRNA SNHG12}

SNHG12 is also involved in regulation of neuroinflammation. SNHG12 was found upregulated in the in vitro model of IS. Silencing of SNHG12 increased neuronal apoptosis via enhanced expression of pro-apoptotic Bcl-2 family members. Moreover, silencing of SNHG12 also resulted in increased levels of pro-inflammatory cytokines such as IL-6 or Eselectin. Thus, SNHG12 shows anti-apoptotic and antiinflammatory roles in ischemic conditions. Finally, the study reported that knockdown of SNHG12 is associated with a lower ratio of $\mathrm{pAkt} / \mathrm{Akt}$ proteins, suggesting that Akt signaling pathway is involved in neuronal cell survival as activation of this axis reduces the survival [86]. The study shows that lncRNA SNHG12 is a promising candidate for stroke treatment in the future; however, further in vivo and human studies are needed.

\section{LncRNA TUG1}

Microglia play an important role in neuroinflammation. LncRNA TUG1 was upregulated in microglial cells and acted as a sponge for miR-145a-5p, negatively regulating its expression. Silencing TUG1 modified the phenotype of microglia (M1-like to M2-like) and reduced pro-inflammatory cytokines which promoted production of anti-inflammatory cytokines, thus increasing cell survival. Additionally, the OGD/Rinduced activation of the NF- $\mathrm{KB}$ pathway was halted by the knockout of TUG1. A competitive interaction between TUG1 and miR-145a-5p was implied and it was shown that the miR$145 \mathrm{a}-5 \mathrm{p}$ inhibition abrogated the NF- $\mathrm{KB}$ inactivating effect of TUG1 knockout. Thus, the NF-KB cascade is involved in TUG1/mir-145a-5p-mediated inflammatory response. Hence, it is assumed that TUG1 can synchronize microglia and assembly of inflammatory cytokines shortly following OGD insult [87]. LncRNA TUG1 regulates microglia that are present in brain lesions. Essentially, microglia have two phenotypes, M1 which is pro-inflammatory and M2, which produces anti-inflammatory particles. TUG1 silencing promotes the differentiation to the M2 phenotype. It was shown that the M2 state is able to promote neuronal repair and regeneration via phagocytosis of harmful substances and debris in the injured area [88] (Fig. 3i).

Collectively, these studies show promising approaches for future IS treatment by targeting neuroinflammation. LncRNAs are able to decrease post-ischemic inflammation through targeting inflammasome formation and microglia phenotype change, important processes contributing to neural inflammation after IS [89]. Thus, all of the mentioned IncRNAs seem to be promising therapeutic approaches in IS and are therefore worthy of further clinical research (Fig. 5) (Table 1). 


\section{Future Perspectives of IncRNAs in IS Treatment}

Nowadays, available treatment methods of IS are limited and associated with possible detrimental effects such as reperfusion brain injury, and thus identification of new therapeutic approaches is urgent. Since lncRNAs are unquestionably abundant in the central nervous system and are involved in cerebral pathophysiological processes, understanding their molecular background and link with IS is essential. Numerous lncRNAs are also associated with specific neuroanatomical regions, suggesting their potentially specific functional role in the nervous system [90]. Moreover, loss and gain of function studies showed that lncRNAs may contribute to secondary damage after brain injury [91]. The abovementioned studies show that regulation of lncRNAs may exert pro-angiogenic, neuroregenerative, anti-apoptotic, and anti-inflammatory effects in injured brain tissue. Importantly, recent data demonstrates that treatment which targets non-coding RNAs or uses their molecules might be an effective approach in IS [92]. A number of studies report that miRNAs-inhibitors/mimics ameliorate IS brain injury and improve recovery and prognosis. Nevertheless, similar data about lncRNAs is limited. Current knowledge about lnRNA's influence on IS is still not sufficient to support their use as therapeutic approaches. There is a lack of human studies and clinical trials involving IncRNAs in IS treatment. Therefore, more studies are necessary to clarify their role in this specific context as current studies showed that lncRNAs are promising approaches for ischemia.

\section{Advantages and Limitations of Using IncRNAs as Therapeutics in IS}

One of the main advantages offered by using lncRNAs in IS treatment is their possibility to interact with miRNAs and coordinate their functions. One of the most unique regulatory roles is that lncRNAs are able to act as sponges for miRNAs. Therefore lncRNAs function as competing genes for miRNAs and inhibit/stimulate the modulatory role of miRNAs as targeted mRNAs. Moreover, as lncRNAs can regulate miRNA expression levels, it gives possible opportunities to use miRNAs as soluble biomarkers of therapeutic response to specific lncRNAs. Additionally, lncRNA may exert a more powerful effect on specific biological processes compared to miRNA. Some of the examples of structural and functional regulatory mechanisms of IncRNAs include regulation of mRNAs stability for protein synthesis, chromatin remodeling, cell cycle control, splicing regulation, targeting specific DNA sequences, as well as earlier mentioned miRNA regulation [93-95]. A number of studies confirm that the gene expression and regulation by lncRNAs are far more complex and extensive than that of miRNAs [96, 97]. Moreover, there is an increasing number of publications and novel searching methods allowing for further lncRNA investigation as well as IncRNA-miRNA interactions, including in silico prediction by using bioinformatic analysis $[94,95]$. Besides, IncRNAs expression is highly specific for tissue, disease, and developmental stages [7], suggesting that therapeutic interference targeting lncRNAs might be more applicable than miRNAs targeting which are rarely specific for a single tissue [98]. On the other hand, the use of lncRNAs in clinical practice faces many limitations: (i) lack of human studies; (ii) all of those lncRNAs discussed in this review need further confirmation; (iii) individual molecules examined in patients with IS such as MALAT1 and Rian are not specific only to IS entity; (iv) although similar expression of single lncRNAs in ischemia has been confirmed independently by many researchers, describing and validation of promising therapeutics signature patterns in IS remain challenging (Fig. 5). Therefore, much work is still needed in this field. Nevertheless, the potential clinical impact is worth the investment.

\section{Conclusions}

LncRNAs are promising targets in IS treatment, especially in the modification of reperfusion injury, which can exacerbate deficits caused by the initial ischemia [99]. Although the underlying mechanism of $\mathrm{I} / \mathrm{R}$ injury is not fully understood, previous studies emphasized the pivotal role of mitochondrial dysfunction, excessive calcium accumulation, and glutamate excitotoxicity in neuronal apoptosis. In our review, we demonstrated that lncRNAs are able to indirectly regulate serine/ threonine kinases (CaMKII, DAPK1) involved in the abovementioned neuronal cell injury pathomechanism. Thus, IncRNAs seem to have therapeutic potential on a cellular level. As it was shown, IncRNAs are also part of neuronal signaling pathways (lncRNA-miRNA-mRNA) responsible for apoptosis and IS severity. Additionally, lncRNAs are able to regulate RNA transcripts by acting as ceRNAs of miRNAs. In many studies this crosstalk could be a potential explanation of how IncRNAs control I/R injury. Moreover, in several studies, the expression rate of lncRNAs fluctuated in a specific manner and correlated with neurological deficit. This may indicate that fast lncRNA intervention may potentially limit the range of injury. Many studies have validated the crucial impact of IncRNA modification in autophagy, angiogenesis, and oxidative stress in IS. We should emphasize the importance of the lncRNAs MALAT1, H19, and SNHG12 which are involved in those processes simultaneously, thus potentially demonstrating increased therapeutic effectiveness. Furthermore, in the case of autophagy in IS, it should be noticed that autophagy has a positive effect only in the initial phases of ischemia. However, prolonged autophagy is fatal for brain tissue. Thus, IncRNAs which are able to target autophagy in IS should be highly specific for the phase of reperfusion. 
Additionally, the role of lncRNAs in regulating postischemic inflammation should be emphasized, as many of them are able to reduce the level of pro-inflammatory cytokines. LncRNA MEG3 is able to regulate inflammasomes, a source of inflammatory molecules that are able to induce pyroptosis, an alternative neuronal cell death pathway. Moreover, lncRNA TUG1 is able to change the microglia phenotype from pro-inflammatory to anti-inflammatory, leading to enhanced neuronal repair. Last but not least, IncRNAs are involved in neurogenesis and play an important role in post-ischemic recovery. Silencing of lncRNA MEG3 increased the levels of BDNF, NGF, and bFGF, all being pivotal factors in neuronal repair processes, including axonal growth and proliferation of neuron progenitors.

Collectively, various pathological mechanisms are involved in exacerbating $\mathrm{I} / \mathrm{R}$ injuries, of which the underlying processes are still not fully understood. LncRNAs can be clinically useful in IS treatment on multiple stages. In Fig. 5, miRNAs/lncRNAs, their target genes, and processes that are involved in pathophysiology of IS were summarized based on the published data. According to this network, we can conclude that IncRNA MALAT1, SNHG12, MEG3, and H19 seem to be the most promising IncRNAs as they can regulate at least three different processes.

Acknowledgments This article was written as a part of cooperation of the international scientific group I-COMET (International Cardiovascular and Cardiometabolic Research Team).

Authors' Contributions MW and CE contributed to the data collection and elaboration, writing, and approval of the manuscript and are guarantors of the article. JJP, LS, EJ, PC, TP, AC, DMG, and ZW contributed to the discussion and writing and approval of manuscript. DMG and $\mathrm{AC}$ contributed to the valuable revision of the manuscript. CE and ZW contributed valuably to the graphical designs. The corresponding author attests that all listed authors meet authorship criteria and that no others meeting the criteria have been omitted.

Funding The work was supported financially as part of the research grant 'OPUS' from National Science Center, Poland (grant number 2018/31/B/ NZ7/01137) and 'Preludium' from the National Science Center, Poland (grant number 2017/25/N/NZ5/00545).

Data Availability Not applicable.

\section{Compliance with Ethical Standards}

Conflict of Interest The authors have no conflicts of interest to declare that are relevant to the content of this article.

Ethics Approval Not applicable.

Consent to Participate Not applicable.

Consent for Publication Not applicable.

Code Availability Not applicable.
Open Access This article is licensed under a Creative Commons Attribution 4.0 International License, which permits use, sharing, adaptation, distribution and reproduction in any medium or format, as long as you give appropriate credit to the original author(s) and the source, provide a link to the Creative Commons licence, and indicate if changes were made. The images or other third party material in this article are included in the article's Creative Commons licence, unless indicated otherwise in a credit line to the material. If material is not included in the article's Creative Commons licence and your intended use is not permitted by statutory regulation or exceeds the permitted use, you will need to obtain permission directly from the copyright holder. To view a copy of this licence, visit http://creativecommons.org/licenses/by/4.0/.

\section{References}

1. Eyileten C, Wicik Z, De Rosa S et al (2018) MicroRNAs as diagnostic and prognostic biomarkers in ischemic stroke-a comprehensive review and bioinformatic analysis. Cells 7. https://doi.org/10. 3390/cells 7120249

2. You D, You H (2019) Repression of long non-coding RNA MEG3 restores nerve growth and alleviates neurological impairment after cerebral ischemia-reperfusion injury in a rat model. Biomed Pharmacother 111:1447-1457

3. Wang H, Zheng X, Jin J, Zheng L, Guan T, Huo Y, Xie S, Wu Y et al (2020) LncRNA MALAT1 silencing protects against cerebral ischemia-reperfusion injury through miR-145 to regulate AQP4. J Biomed Sci 27:40

4. Li Z, Li J, Tang N (2017) Long noncoding RNA Malat1 is a potent autophagy inducer protecting brain microvascular endothelial cells against oxygen-glucose deprivation/reoxygenation-induced injury by sponging miR-26b and upregulating ULK2 expression. Neuroscience 354:1-10

5. Quinn JJ, Chang HY (2016) Unique features of long non-coding RNA biogenesis and function. Nat Rev Genet 17:47-62

6. Jing H, Liu L, Jia Y, Yao H, Ma F (2019) Overexpression of the long non-coding RNA Oprm1 alleviates apoptosis from cerebral ischemia-reperfusion injury through the Oprm1/miR-155/GATA3 axis. Artif Cells Nanomed Biotechnol 47:2431-2439

7. Paraskevopoulou MD, Hatzigeorgiou AG (2016) Analyzing MiRNA-LncRNA interactions. Methods Mol Biol 1402:271-286

8. Pordzik J, Jakubik D, Jarosz-Popek J, Wicik Z, Eyileten C, de Rosa S, Indolfi C, Siller-Matula JM et al (2019) Significance of circulating microRNAs in diabetes mellitus type 2 and platelet reactivity: bioinformatic analysis and review. Cardiovasc Diabetol 18:113

9. Pordzik J, Pisarz K, De Rosa S et al (2018) The potential role of platelet-related microRNAs in the development of cardiovascular events in high-risk populations, including diabetic patients: a review. Front Endocrinol 9:74

10. Sabatino J, Wicik Z, De Rosa S et al (2019) MicroRNAs fingerprint of bicuspid aortic valve. J Mol Cell Cardiol 134:98-106

11. Arun G, Diermeier SD, Spector DL (2018) Therapeutic targeting of long non-coding RNAs in cancer. Trends Mol Med 24:257-277

12. Chen X, Li C, Li J, Sheng L, Liu X (2019) Upregulation of miR1306-5p decreases cerebral ischemia/reperfusion injury by targeting BIK. Biosci Biotechnol Biochem 83:2230-2237

13. Xiong W, Wu Y, Xian W, Song L, Hu L, Pan S, Liu M, Yao S et al (2018) DAPK1-ERK signal mediates oxygen glucose deprivation reperfusion induced apoptosis in mouse N2a cells. J Neurol Sci 387:210-219

14. Xu Q, Deng F, Xing Z, Wu Z, Cen B, Xu S, Zhao Z, Nepomuceno $\mathrm{R}$ et al (2016) Long non-coding RNA C2dat1 regulates CaMKII $\delta$ expression to promote neuronal survival through the NF- $\mathrm{K} B$ 
signaling pathway following cerebral ischemia. Cell Death Dis 7: e2173-e2173

15. Rostas JAP, Spratt NJ, Dickson PW, Skelding KA (2017) The role of Ca-calmodulin stimulated protein kinase II in ischaemic stroke a potential target for neuroprotective therapies. Neurochem Int 107: $33-42$

16. Hajimohammadreza I, Probert AW, Coughenour LL, Borosky SA, Marcoux FW, Boxer PA, Wang KK (1995) A specific inhibitor of calcium/calmodulin-dependent protein kinase-II provides neuroprotection against NMDA- and hypoxia/hypoglycemia-induced cell death. J Neurosci 15:4093-4101

17. Ashpole NM, Song W, Brustovetsky T, Engleman EA, Brustovetsky N, Cummins TR, Hudmon A (2012) Calcium/ calmodulin-dependent protein kinase II (CaMKII) inhibition induces neurotoxicity via dysregulation of glutamate/calcium signaling and hyperexcitability. J Biol Chem 287:8495-8506

18. Feng M, Zhu X, Zhuo C (2020) H19/miR-130a-3p/DAPK1 axis regulates the pathophysiology of neonatal hypoxic-ischemia encephalopathy. Neurosci Res. https://doi.org/10.1016/j.neures. 2020.03.005

19. Wei R, Zhang L, Hu W, Wu J, Zhang W (2019) Long non-coding RNA AK038897 aggravates cerebral ischemia/reperfusion injury via acting as a ceRNA for miR-26a-5p to target DAPK1. Exp Neurol 314:100-110

20. Tu W, Xu X, Peng L, Zhong X, Zhang W, Soundarapandian MM, Belal C, Wang M et al (2010) DAPK1 interaction with NMDA receptor NR2B subunits mediates brain damage in stroke. Cell 140:222-234

21. Yao P, Li Y-L, Chen Y, Shen W, Wu KY, Xu WH (2020) Overexpression of long non-coding RNA Rian attenuates cell apoptosis from cerebral ischemia-reperfusion injury via Rian/miR144-3p/GATA3 signaling. Gene 737:144411

22. Matsui Y, Takagi H, Qu X, Abdellatif M, Sakoda H, Asano T, Levine B, Sadoshima J (2007) Distinct roles of autophagy in the heart during ischemia and reperfusion: roles of AMP-activated protein kinase and Beclin 1 in mediating autophagy. Circ Res 100: 914-922

23. He Q-W, Li Q, Jin H-J, Zhi F, Suraj B, Zhu YY, Xia YP, Mao L et al (2016) MiR-150 regulates poststroke cerebral angiogenesis via vascular endothelial growth factor in rats. CNS Neurosci Ther 22: 507-517

24. Adamczak SE, de Rivero Vaccari JP, Dale G, Brand FJ III, Nonner D, Bullock MR, Dahl GP, Dietrich WD et al (2014) Pyroptotic neuronal cell death mediated by the AIM2 inflammasome. J Cereb Blood Flow Metab 34:621-629

25. Zhao M, Wang J, Xi X, Tan N, Zhang L (2018) SNHG12 promotes angiogenesis following ischemic stroke via regulating miR-150/ VEGF pathway. Neuroscience 390:231-240

26. Liang J, Wang Q, Li J-Q, Guo T, Yu D (2020) Long non-coding RNA MEG3 promotes cerebral ischemia-reperfusion injury through increasing pyroptosis by targeting miR-485/AIM2 axis. Exp Neurol 325:113139

27. Eyileten C, Mirowska-Guzel D, Milanowski L, Zaremba M, Rosiak M, Cudna A, Kaplon-Cieslicka A, Opolski G et al (2019) Serum brain-derived neurotrophic factor is related to platelet reactivity and metformin treatment in adult patients with type 2 diabetes mellitus. Can J Diabetes 43:19-26

28. Mirowska-Guzel D (2009) The role of neurotrophic factors in the pathology and treatment of multiple sclerosis. Immunopharmacol Immunotoxicol 31:32-38

29. Yu S-Y, Dong B, Fang Z-F, Hu XQ, Tang L, Zhou SH (2018) Knockdown of lncRNA AK139328 alleviates myocardial ischaemia/reperfusion injury in diabetic mice via modulating miR-204-3p and inhibiting autophagy. J Cell Mol Med 22:48864898
30. Suofu Y, Wang X, He Y, Li F, Zhang Y, Carlisle DL, Friedlander RM (2020) Mir-155 knockout protects against ischemia/ reperfusion-induced brain injury and hemorrhagic transformation. Neuroreport 31:235-239

31. Wang K, Liu C-Y, Zhou L-Y, Wang JX, Wang M, Zhao B, Zhao WK, Xu SJ et al (2015) APF IncRNA regulates autophagy and myocardial infarction by targeting miR-188-3p. Nat Commun 6 : 6779

32. Wang S, Han X, Mao Z, Xin Y, Maharjan S, Zhang B (2019) MALAT1 lncRNA induces autophagy and protects brain microvascular endothelial cells against oxygen-glucose deprivation by binding to miR-200c-3p and upregulating SIRT1 expression. Neuroscience 397:116-126

33. Yan H, Rao J, Yuan J, Gao L, Huang W, Zhao L, Ren J (2017) Long non-coding RNA MEG3 functions as a competing endogenous RNA to regulate ischemic neuronal death by targeting miR21/PDCD4 signaling pathway. Cell Death Dis 8:3211

34. Mirowska-Guzel D, Litwin T, Gromadzka G, Czlonkowski A, Czlonkowska A (2013) Influence of BDNF polymorphisms on Wilson's disease susceptibility and clinical course. Metab Brain Dis 28:447-453

35. Wu Z, Wu P, Zuo X, Yu N, Qin Y, Xu Q, He S, Cen B et al (2017) Erratum to: LncRNA-N1LR enhances neuroprotection against ischemic stroke probably by inhibiting p53 phosphorylation. Mol Neurobiol 54:7686-7688

36. Guo D, Ma J, Yan L, Li T, Li Z, Han X, Shui S (2017) Downregulation of lncRNA MALAT1 attenuates neuronal cell death through suppressing Beclin1-dependent autophagy by regulating Mir-30a in cerebral ischemic stroke. Cell Physiol Biochem 43: 182-194

37. Yang Y, Duan W, Li Y, Yan J, Yi W, Liang Z, Wang N, Yi D et al (2013) New role of silent information regulator 1 in cerebral ischemia. Neurobiol Aging 34:2879-2888

38. Shi R, Weng J, Zhao L, Li XM, Gao TM, Kong J (2012) Excessive autophagy contributes to neuron death in cerebral ischemia. CNS Neurosci Ther 18:250-260

39. Liu C, Xu P, Chen D et al (2013) Roles of autophagy-related genes Beclin-1 and LC3 in the development and progression of prostate cancer and benign prostatic hyperplasia. Biomed Rep 1:855-860

40. Deng Y, Chen D, Gao F, Lv H, Zhang G, Sun X, Liu L, Mo D et al (2020) Silencing of long non-coding RNA GAS5 suppresses neuron cell apoptosis and nerve injury in ischemic stroke through inhibiting DNMT3B-dependent MAP4K4 methylation. Transl Stroke Res 11:950-966. https://doi.org/10.1007/s12975-01900770-3

41. Tsarovina K, Reiff T, Stubbusch J, Kurek D, Grosveld FG, Parlato R, Schutz G, Rohrer H (2010) The Gata3 transcription factor is required for the survival of embryonic and adult sympathetic neurons. J Neurosci 30:10833-10843

42. Wang K, Liu F, Zhou L-Y, Long B, Yuan SM, Wang Y, Liu CY, Sun $\mathrm{T}$ et al (2014) The long noncoding RNA CHRF regulates cardiac hypertrophy by targeting miR-489. Circ Res 114:13771388

43. Gai H-Y, Wu C, Zhang Y, Wang D (2019) Long non-coding RNA CHRF modulates the progression of cerebral ischemia/reperfusion injury via miR-126/SOX6 signaling pathway. Biochem Biophys Res Commun 514:550-557

44. Carino A, De Rosa S, Sorrentino S et al (2016) Modulation of circulating microRNAs levels during the switch from clopidogrel to ticagrelor. Biomed Res Int 2016:3968206

45. Fateh-Moghadam S, Htun P, Tomandl B, Sander D, Stellos K, Geisler T, Langer H, Walton K et al (2007) Hyperresponsiveness of platelets in ischemic stroke. Thromb Haemost 97:974-978

46. Cai J, Shangguan S, Li G, Cai Y, Chen Y, Ma G, Miao Z, Liu L et al (2019) Knockdown of lncRNA Gm11974 protect against cerebral 
ischemic reperfusion through miR-766-3p/NR3C2 axis. Artif Cells Nanomed Biotechnol 47:3847-3853

47. Ozoner B, Yuceli S, Aydin S, Yazici GN, Sunar M, Arslan YK, Coban TA, Suleyman H (2019) Effects of pycnogenol on ischemia/ reperfusion-induced inflammatory and oxidative brain injury in rats. Neurosci Lett 704:169-175

48. Zhang X, Yan H, Yuan Y, Gao J, Shen Z, Cheng Y, Shen Y, Wang RR et al (2013) Cerebral ischemia-reperfusion-induced autophagy protects against neuronal injury by mitochondrial clearance. Autophagy 9:1321-1333

49. Sprott D, Poitz DM, Korovina I, Ziogas A, Phieler J, Chatzigeorgiou A, Mitroulis I, Deussen A et al (2019) Endothelial-specific deficiency of ATG5 (autophagy protein 5) attenuates ischemia-related angiogenesis. Arterioscler Thromb Vasc Biol 39:1137-1148

50. Kulkarni VV, Maday S (2018) Compartment-specific dynamics and functions of autophagy in neurons. Dev Neurobiol 78:298-310

51. Wang P, Shao B-Z, Deng Z, Chen S, Yue Z, Miao CY (2018) Autophagy in ischemic stroke. Prog Neurobiol 163-164:98-117

52. Wang P, Guan Y-F, Du H et al (2012) Induction of autophagy contributes to the neuroprotection of nicotinamide phosphoribosyltransferase in cerebral ischemia. Autophagy 8:7787

53. Liu C-Y, Zhang Y-H, Li R-B, Zhou LY, An T, Zhang RC, Zhai M, Huang Y et al (2018) LncRNA CAIF inhibits autophagy and attenuates myocardial infarction by blocking p53-mediated myocardin transcription. Nat Commun 9:29

54. Yao X, Yao R, Huang F, Yi J (2019) LncRNA SNHG12 as a potent autophagy inducer exerts neuroprotective effects against cerebral ischemia/reperfusion injury. Biochem Biophys Res Commun 514: 490-496

55. Li Y, Guo S, Liu W, Jin T, Li X, He X, Zhang X, Su H et al (2019) Silencing of SNHG12 enhanced the effectiveness of MSCs in alleviating ischemia/reperfusion injuries via the $\mathrm{PI} 3 \mathrm{~K} / \mathrm{AKT} / \mathrm{mTOR}$ signaling pathway. Front Neurosci 13:645

56. Wang J, Cao B, Han D, Sun M, Feng J (2017) Long non-coding RNA H19 induces cerebral ischemia reperfusion injury via activation of autophagy. Aging Dis 8:71-84

57. Yu S, Yu M, He X, Wen L, Bu Z, Feng J (2019) KCNQ1OT1 promotes autophagy by regulating miR-200a/FOXO3/ATG7 pathway in cerebral ischemic stroke. Aging Cell 18:e12940

58. Puyal J, Vaslin A, Mottier V, Clarke PGH (2009) Postischemic treatment of neonatal cerebral ischemia should target autophagy. Ann Neurol 66:378-389

59. Wang P, Xu T-Y, Wei K, Guan YF, Wang X, Xu H, Su DF, Pei G et al (2014) ARRB1/ $\beta$-arrestin-1 mediates neuroprotection through coordination of BECN1-dependent autophagy in cerebral ischemia. Autophagy 10:1535-1548

60. Ruan L, Wang B, ZhuGe Q, Jin K (2015) Coupling of neurogenesis and angiogenesis after ischemic stroke. Brain Res 1623:166-173

61. Wang C, Qu Y, Suo R, Zhu Y (2019) Long non-coding RNA MALAT1 regulates angiogenesis following oxygen-glucose deprivation/reoxygenation. J Cell Mol Med 23:2970-2983

62. Gao C, Zhang C-C, Yang H-X, Hao Y-N (2020) MALAT1 protected the angiogenesis function of human brain microvascular endothelial cells (HBMECs) under oxygen glucose deprivation/reoxygenation (OGD/R) challenge by interacting with miR-205-5p/ VEGFA pathway. Neuroscience 435:135-145

63. Kalogeris T, Bao Y, Korthuis RJ (2014) Mitochondrial reactive oxygen species: a double edged sword in ischemia/reperfusion vs preconditioning. Redox Biol 2:702-714

64. Pantazi E, Zaouali MA, Bejaoui M, Folch-Puy E, Ben Abdennebi H, Roselló-Catafau J (2013) Role of sirtuins in ischemiareperfusion injury. World J Gastroenterol 19:7594-7602

65. Yin W-L, Yin W-G, Huang B-S, Wu L-X (2019) LncRNA SNHG12 inhibits miR-199a to upregulate SIRT1 to attenuate cerebral ischemia/reperfusion injury through activating AMPK signaling pathway. Neurosci Lett 690:188-195

66. De Rosa S, Eposito F, Carella C et al (2018) Transcoronary concentration gradients of circulating microRNAs in heart failure. Eur J Heart Fail 20:1000-1010

67. Zeng J, Zhu L, Liu J et al (2019) Metformin protects against oxidative stress injury induced by ischemia/reperfusion via regulation of the lncRNA-H19/miR-148a-3p/Rock2 axis. Oxidative Med Cell Longev 2019:8768327

68. Mirowska-Guzel D, Gromadzka G, Czlonkowski A, Czlonkowska A (2012) BDNF -270 C $>$ T polymorphisms might be associated with stroke type and BDNF -196 G $>$ A corresponds to early neurological deficit in hemorrhagic stroke. J Neuroimmunol 249:71-75

69. Mirowska-Guzel D, Gromadzka G, Seniow J, Lesniak M, Bilik M, Waldowski K, Gruchala K, Czlonkowski A et al (2013) Association between BDNF-196 G>A and BDNF-270 C $>$ T polymorphisms, BDNF concentration, and rTMS-supported long-term rehabilitation outcome after ischemic stroke. NeuroRehabilitation 32:573-582

70. Mirowska-Guzel D, Gromadzka G, Mendel T, Janus-Laszuk B, Dzierka J, Sarzynska-Dlugosz I, Czlonkowski A, Czlonkowska A (2014) Impact of BDNF -196 G>A and BDNF -270 C $>$ T polymorphisms on stroke rehabilitation outcome: sex and age differences. Top Stroke Rehabil 21(Suppl 1):S33-S41

71. Eyileten C, Sharif L, Wicik Z, Jakubik D, Jarosz-Popek J, Soplinska A, Postula M, Czlonkowska A et al (2020) The relation of the brain-derived neurotrophic factor with microRNAs in neurodegenerative diseases and ischemic stroke. Mol Neurobiol. https://doi.org/10.1007/s12035-020-02101-2

72. Cruz Y, Lorea J, Mestre H, Kim-Lee JH, Herrera J, Mellado R, Gálvez V, Cuellar L et al (2015) Copolymer-1 promotes neurogenesis and improves functional recovery after acute ischemic stroke in rats. PLoS One 10:e0121854

73. Eyileten C, Kaplon-Cieslicka A, Mirowska-Guzel D et al (2017) Antidiabetic effect of brain-derived neurotrophic factor and its association with inflammation in type 2 diabetes mellitus. J Diabetes Res 2017:2823671

74. Eyileten C, Zaremba M, Janicki PK, Rosiak M, Cudna A, KapłonCieślicka A, Opolski G, Filipiak KJ et al (2016) Serum brainderived neurotrophic factor is related to platelet reactivity but not to genetic polymorphisms within BDNF encoding gene in patients with type 2 diabetes. Med Sci Monit 22:69-76

75. Kokaia Z, Thored P, Arvidsson A, Lindvall O (2006) Regulation of stroke-induced neurogenesis in adult brain-recent scientific progress. Cereb Cortex 16(Suppl 1):i162-i167

76. Hawkins KE, DeMars KM, Alexander JC et al (2017) Targeting resolution of neuroinflammation after ischemic stroke with a lipoxin A4 analog: protective mechanisms and long-term effects on neurological recovery. Brain Behav 7:e0688

77. Ng S-Y, Bogu GK, Soh BS, Stanton LW (2013) The long noncoding RNA RMST interacts with SOX2 to regulate neurogenesis. Mol Cell 51:349-359

78. Hou X-X, Cheng H (2018) Long non-coding RNA RMST silencing protects against middle cerebral artery occlusion (MCAO)-induced ischemic stroke. Biochem Biophys Res Commun 495:2602-2608

79. Sims NR, Yew WP (2017) Reactive astrogliosis in stroke: contributions of astrocytes to recovery of neurological function. Neurochem Int 107:88-103

80. Pekny M, Wilhelmsson U, Tatlisumak T, Pekna M (2019) Astrocyte activation and reactive gliosis-a new target in stroke? Neurosci Lett 689:45-55

81. Guzel T, Mech K, Wroński M et al (2020) Brain-derived neurotrophic factor in gastroenterology oncology - short review of current literature. Ann Agric Environ Med. https://doi.org/10.26444/ aaem $/ 122628$ 
82. Lénárt N, Brough D, Dénes Á (2016) Inflammasomes link vascular disease with neuroinflammation and brain disorders. J Cereb Blood Flow Metab 36:1668-1685

83. Gao L, Dong Q, Song Z, Shen F, Shi J, Li Y (2017) NLRP3 inflammasome: a promising target in ischemic stroke. Inflamm Res 66:17-24

84. Hong P, Gu R-N, Li F-X, Xiong XX, Liang WB, You ZJ, Zhang HF (2019) NLRP3 inflammasome as a potential treatment in ischemic stroke concomitant with diabetes. J Neuroinflammation 16:121

85. Zhang X, Tang X, Liu K, Hamblin MH, Yin KJ (2017) Long noncoding RNA Malat1 regulates cerebrovascular pathologies in ischemic stroke. J Neurosci 37:1797-1806

86. Cheng Y, Jiang Y, Sun Y, Jiang H (2019) The role of long noncoding RNA SNHG12 in neuroprotection following cerebral ischemic injury. Neuroreport 30:945-952

87. Wang H, Liao S, Li H, Chen Y, Yu J (2019) Long non-coding RNA TUG1 sponges Mir-145a-5p to regulate microglial polarization after oxygen-glucose deprivation. Front Mol Neurosci 12:215

88. Wang J, Xing H, Wan L et al (2018) Treatment targets for M2 microglia polarization in ischemic stroke. Biomed Pharmacother 105:518-525

89. Zheng D, Liwinski T, Elinav E (2020) Inflammasome activation and regulation: toward a better understanding of complex mechanisms. Cell Discov 6:36

90. Mercer TR, Dinger ME, Sunkin SM, Mehler MF, Mattick JS (2008) Specific expression of long noncoding RNAs in the mouse brain. Proc Natl Acad Sci U S A 105:716-721

91. He D, Wang J, Lu Y, Deng Y, Zhao C, Xu L, Chen Y, Hu YC, Zhou W, Lu QR (2017) lncRNA Functional networks in oligodendrocytes reveal stage-specific myelination control by an lncOL1/ Suz12 complex in the CNS. Neuron 93(2):362-378. https://doi.org/ 10.1016/j.neuron.2016.11.044
92. Wang S-W, Liu Z, Shi Z-S (2018) Non-coding RNA in acute ischemic stroke: mechanisms, biomarkers and therapeutic targets. Cell Transplant 27:1763-1777

93. Li Z, Zhao W, Wang M, Zhou X (2019) The role of long noncoding RNAs in gene expression regulation. In: Vlachakis D (ed) Gene expression profiling in cancer. IntechOpen. https://doi.org/10. 5772/intechopen.81773

94. Sun B, Liu C, Li H, Zhang L, Luo G, Liang S, Lü M (2020) Research progress on the interactions between long non-coding RNAs and microRNAs in human cancer (review). Oncol Lett 19: 595-605

95. Perron U, Provero P, Molineris I (2017) In silico prediction of lncRNA function using tissue specific and evolutionary conserved expression. BMC Bioinformatics 18:29-39

96. Mercer TR, Dinger ME, Mattick JS (2009) Long non-coding RNAs: insights into functions. Nat Rev Genet 10:155-159

97. Wilusz JE, Sunwoo H, Spector DL (2009) Long noncoding RNAs: functional surprises from the RNA world. Genes Dev 23:1494 1504

98. Ludwig N, Leidinger P, Becker K, Backes C, Fehlmann T, Pallasch C, Rheinheimer S, Meder B et al (2016) Distribution of miRNA expression across human tissues. Nucleic Acids Res 44:3865-3877

99. Pan J, Konstas A-A, Bateman B, Ortolano GA, Pile-Spellman J (2007) Reperfusion injury following cerebral ischemia: pathophysiology, MR imaging, and potential therapies. Neuroradiology 49: 93-102

Publisher's Note Springer Nature remains neutral with regard to jurisdictional claims in published maps and institutional affiliations. 